\title{
Theory of Superfluids with Population Imbalance: Finite Temperature and BCS-BEC Crossover Effects
}

\author{
Qijin Chen, Yan He, Chih-Chun Chien and K. Levin \\ James Franck Institute and Department of Physics, University of Chicago, Chicago, Illinois 60637
}

(Dated: 22nd August 2018)

\begin{abstract}
In this paper we present a very general theoretical framework for addressing fermionic superfluids over the entire range of BCS to Bose Einstein condensation (BEC) crossover in the presence of population imbalance or spin polarization. Our emphasis is on providing a theory which reduces to the standard zero temperature mean field theories in the literature, but necessarily includes pairing fluctuation effects at non-zero temperature within a consistent framework. Physically, these effects are associated with the presence of pre-formed pairs (or a fermionic pseudogap) in the normal phase, and pair excitations of the condensate, in the superfluid phase. We show how this finite $T$ theory of fermionic pair condensates bears many similarities to the condensation of point bosons. In the process we examine three different types of condensate: the usual breached pair or Sarma phase and both the one and two plane wave Larkin- Ovchinnikov, Fulde-Ferrell (LOFF) states. The last of these has been discussed in the literature albeit only within a Landau-Ginzburg formalism, generally valid near $T_{c}$. Here we show how to arrive at the two plane wave LOFF state in the ground state as well as at general temperature $T$.

PACS numbers: 03.75.Hh, 03.75.Ss, 74.20.-z
\end{abstract}

\section{INTRODUCTION}

The subject of superfluidity in ultracold trapped fermionic gases is an exciting field [1, 2, 3, 4, 5, 6, 7, 8], not only for its implications in atomic physics, but also because there are important implications for condensed matter systems, including perhaps high temperature superconductors [9, 10]. There are two important aspects which are particularly notable about these systems, from the perspective of the present paper. They can be tuned in various ways which are not available to naturemade superconductors. Thus, one can study the entire regime from BCS to Bose-Einstein condensation (BEC), simply by the application of a magnetic field in concert with a Feshbach resonance. Moreover, one can vary the concentrations of the two spin species arbitrarily [11, 12, 13]; in a fashion, this simulates the application of a magnetic Zeeman field. This latter tuneability has important implications for other subdisciplines in physics such as dense QCD and (isospin asymmetric) nuclear matter [14, 15, 16]. Equally important is the fact that there is a rich collection of experimental data from two different atomic physics groups [11, 12, 13] on ${ }^{6} \mathrm{Li}$ gases near unitarity, with which one can compare various theoretical results.

The goal of this paper is to present an overarching theoretical framework for dealing with fermionic gases over the entire range of BCS to BEC crossover and the entire range of temperature, as well as the entire range of population imbalance. The foundations of this theory lie with the initial observation of Eagles [17] and of Leggett [18] that the BCS-like wavefunction has a much greater generality than was originally recognized at the time of its proposal. It is capabable of describing both BCS and BEC like systems, providing the pairing attraction is tuneable from arbitrarily weak (BCS) to arbitrarily strong (BEC) and one self consistently solves for the fermionic chemical potential. This mean field-like ground state wave function is also readily generalized to include pop- ulation balance. Indeed, there are at least three well studied phases [19, 20, 21] which have been proposed to accomodate a difference in the population of the two spin species. What we want to stress is that these same mean field theories have a natural extension to finite temperature [9]. This extension will be a focus of the present paper.

At strictly zero temperature, there is a rather extensive literature [22, 23, 24, 25, 26, 27, 28] on these populationimbalanced superfluids and superconductors, including recently the effects of the crossover from BCS to BEC. There have also been some studies at finite $T$, which are at the mean field level and do not include the effect of the non-condensed pairs [29, 30, 31] we consider here. The three most studied phases are the "Sarma"-like [19] or breached pair states in which (as in BCS theory) the condensed pairs have zero net momentum, but polarization can, nevertheless, be introduced. At $T=0$ this state appears to be stable in the deep BEC regime. Additionally, two different phases [21] have been proposed by Larkin and Ovchinnikov and by Fulde and Ferrell (LOFF) in which the condensate has a net momentum of a pre-determined $\mathbf{q}$ or of $\pm \mathbf{q}$. Even more elaborate crystalline lattices of various $\mathbf{q}_{i}$ have also been contemplated [32]. It is believed that these states are more appropriate closer to the BCS side of resonance, although rather little has been determined about the "two-plane wave" LOFF state near $T=0$ or in the presence of crossover effects. Added to this complexity is the possiblity of heterogeneous or phase separated states [20].

In this paper we will present the theoretical formalism for the Sarma and one and two-plane wave LOFF states at zero and finite $T$ as one varies from BCS to BEC. We note that because theories of population imbalanced superfluids are (currently, without exception) based on BCS-Leggett-type [18] ground states, it is important to determine their finite temperature implications within this broad class of ground states, as we do here. Our premise is that the effects of finite $T$, which 
necessarily must be accomodated in any comparison with experiment, must be compatible with the $T=0$ formalism. Indeed, one of the most important effects of temperature is to stabilize the Sarma-like phase. In this way one finds an intermediate temperature superfluid [33, 34, 35, 36], one that exists at $T \neq 0$, but not at $T=0$. Our studies of the two-plane wave LOFF state present new results by extending the current literature away from the Landau-Ginzburg regime (near $T_{c}$ ).

With this class of generalized mean field theories we will show that the effects of temperature enter in a fashion which is strongly reminiscent of Bose gas condensation. Except in the BCS limit, pairs form at higher temperatures, than the (transition) temperature $T_{c}$ at which they condense. Thus we have to distinguish the excitation gap [called $\Delta(T)$ ] from the order parameter [called $\Delta_{s c}(T)$ ]. Alternatively, this means that there is an excitation gap (or pseudogap $\Delta_{p g}^{2}$ ) for fermionic excitations even in the normal phase. It also implies that below $T_{c}$ there will be additional modes of exciting the condensate- via pair excitations. These latter are noncondensed or incoherent pairs with finite center of mass momentum. We have found [34] that in a trapped geometry they are particularly important for providing a mechanism of getting polarization into the gas.

These effects of finite $T$ can be compared with an alternative class of theories in the literature based on work by Nozieres and Schmitt-Rink (NSR) [37]. This approach is known to lack self-consistency [38]. We stress that the finite temperature NSR approach was not designed to be consistent with the standard ground state equations. This observation has also been made in Ref. [39]. Indeed, these latter authors have presented in considerable detail [40] a more complete finite $T$ approach based on the $T_{c}$ calculations of Ref. [37]. A major concern about this class of theories remains to be addressed. Because the gap and the number equations are not treated on an equivalent basis, it is possible that the superfluid density will not consistently vanish at $T_{c}$. At this temperature there has to be a precise, but delicate cancellation of paramagnetic and diamagnetic current contributions, as found in the present theory [41, 42]. In a related fashion, pseudogap effects (associated with the presence of non-condensed pairs) appear, within an NSR-based approach, in the number equation but not in the gap equation.

We begin at the more physical level by stressing the analogy between condensation in this composite boson or fermionic superfluid and condensation in a gas of ideal point bosons. Our theory treats self-consistently two-particle and one-particle Green's functions on an equal footing. Because the physics is so simple and clear, we can fairly readily anticipate the form of the central equations of this BCS-BEC generalization of BCS theory. It is important to stress, however, that these equations can be derived more rigorously from a truncated series of equations of motion for the appropriate Green's functions [43].

There are three principle equations which govern Bose condensation: the vanishing of the bosonic chemical potential at all $T \leq T_{c}$ is the first. Throughout this paper we will refer to this condition as the "BEC condition". It is related to the usual Thouless criterion, but the latter is generally associated only with the temperature $T_{c}$. The second equation is the boson number equation. All "bosons" must be accounted for as either condensed or non-condensed. The third equation is the number of non-condensed "bosons", which are created by thermal excitations. This is determined simply by inserting the known excitation spectrum of the excited pairs or bosons, into the Bose distribution function. With this equation, and the first equation, one can then deduce the number of condensed bosons.

These three central equations for bosons are indicated in Table I, on the far right, for true point bosons, and in the second column for the composite bosons which appear in fermionic superfluids. For these composite bosons the quantity which provides a measure of the "number" of bosons $(N)$ is given by $\Delta^{2}(T)$ (up to a constant coefficient, $Z$ ). This is reasonably easy to see. In the fermionic regime, when the fermionic chemical potential is positive, $\Delta^{2}(T)$ represents the square of the excitation gap. This is the energy which must be supplied to break apart the pairs. Thus, $\Delta^{2}(T)$, in some sense then, reflects the number of pairs. How does one quantitatively establish the appropriate "boson number" for the fermionic case? This is determined via the self consistent gap equation for $\Delta(T)$, which, in turn, is determined using the first condition: that the pair chemical potential is zero at and below $T_{c}$. How does one compute the number of excited pairs? Once the gap equation is interpreted in terms of the appropriate non-condensed pair propagator, then one knows the related excitation spectrum $\Omega_{q}$ of this propagator.

The quantity $Z$ which appears in the last equation of the Table (for the composite bosons) gives the relation between the gap associated with non condensed pairs $\left(\Delta_{p g}^{2}\right)$ and the number of pairs $\left(\sum b\left(\Omega_{q}\right)\right)$. It can be readily calculated in this theory; once one has the non-condensed pair propagator, $Z$ appears as the inverse residue. (Deep in the BEC regime, $Z$ is relatively simple to compute, for here the boson number density approaches the asymptote $n / 2$, where $n$ is the fermion density). More precisely, the total number of bosons in the present case has to be determined self-consistently through the gap equation. It also involves the fermion number equation through the related fermionic chemical potential. In this last context, it should be stressed that there is one important aspect of the fermionic superfluids, which is not apparent in Table I. For BCS-BEC crossover, it is essential to derive the self consistent equation for the fermionic chemical potential; in this problem the fermions are the fundamental statistical entity. This can be readily accomplished within the same framework used to arrive at the gap equation. The vanishing of the pair chemical potential is associated with a particular choice for the pair propagator involving dressed Green's functions. These, in turn, determine the fermionic chemical potential through the fermion number equation. In the next two sections we turn to the gap and number equations, and show through a Green's function formulation, how strongly these two equations are inter-connected.

The rest of the paper is organized as follows. We conclude this section with a summary of the central equations associated with our $T$-matrix scheme. In Sec. III we present a mean-field theory for the Sarma and one-plane-wave LOFF 


\begin{tabular}{|c|c|c|}
\hline & Composite bosons & Point bosons \\
\hline Pair chemical potential & $\begin{array}{c}\mu_{\text {pair }}=0, T \leq T_{c} \\
\text { Leads to BCS gap equation for } \Delta(T)\end{array}$ & $\mu_{B}=0, T \leq T_{c}$ \\
\hline Total "number" of pairs & $\Delta^{2}(T)=\Delta_{s c}^{2}(T)+\Delta_{p g}^{2}(T)$ & $N=N_{0}+N_{T}$ \\
\hline Noncondensed pairs & $Z \Delta_{p g}^{2}=\sum_{\mathbf{q} \neq 0} b\left(\Omega_{q}\right)$ & $N_{T}=\sum_{\mathbf{q} \neq 0} b\left(\Omega_{q}\right)$ \\
\hline
\end{tabular}

Table I: BCS theory by way of BEC analogy. Here we compare condensation in composite and point bosons; $\mu_{B}$ is the bosonic chemical potential, $N_{0}$ is the number of condensed and $N_{T}$ is the number of non-condensed bosons. We define $\mu_{\text {pair }}$ as the chemical potential for the non-condensed pairs. Here $\Delta(T)$ is the total fermionic gap which contains contributions from the non-condensed $\left(\Delta_{p g}^{2}\right)$ and condensed terms $\left(\Delta_{s c}^{2}\right)$. In the strict BCS limit $\Delta_{p g}=0$, so that the order parameter and gap are identical.

state for $T<T_{c}$. Due to the complexity of the two-planewave LOFF state, we dedicate an entire section (Sec III) to its mean-field treatment. In Sec. IV we present a generalization of our $T$-matrix formalism to include pairing fluctuation effects. Section $\nabla$ recapitulates our simple physical picture and Section VI presents our conclusions. Additional, more technical details are given in two Appendices for the one and two plane wave LOFF states.

\section{A. Central Equations of $T$-matrix scheme}

To make contact with the general class of mean field theories (including the Sarma and LOFF states), we introduce a $T$-matrix approximation. This means that we consider the coupled equations between the particles (with propagator $G$ ) and the pairs [with propagator $t(P)]$ and drop all higher terms. This theory does not include direct "boson-boson" interactions, although the pairs do interact indirectly via the fermions, in an averaged or mean field sense. Throughout Sections $\amalg$ and BEC condition noted in the previous section will give the same gap equation we find using standard techniques, such as Bogoliubov diagonalization applied to the linearized mean field Hamiltonian. Here, for all $T \leq T_{c}$, the BEC condition is interpreted as requiring that the pair chemical potential $\mu_{\text {pair }}$ associated with the non-condensed pairs vanish. In Sections and III we will address only the first line of Table I. The second two lines, or sets of equations will be discussed in Section IV]

Within a $T$-matrix scheme, the pair propagator is given by

$$
t^{-1}(P)=U^{-1}+\chi(P)
$$

where $\chi$ is the spin symmetrized pair susceptibility, and $U<0$ is the pairing interaction strength. The function $\chi(P)$ is, in many ways, the most fundamental quantity we introduce in this paper. It provides the basis for obtaining well known (as well as new) results of the zero temperature theory. Moreover, it provides the basis for arriving at a finite temperature description, which appears in Section IV The introduction of spin symmetrization is only important for the case of population imbalance. In earlier literature [9], this complexity did not arise. We will show that one obtains consistent answers between $T$-matrix based approaches and standard mean field theories, provided the components of the pair susceptibility in the presence of population imbalance, defined by

$$
\chi(P)=\frac{1}{2}\left[\chi_{\uparrow \downarrow}(P)+\chi_{\downarrow \uparrow}(P)\right]
$$

is given by the product of one dressed and one bare Green's function

$$
\begin{aligned}
& \chi_{\uparrow \downarrow}(P)=\sum_{K} G_{0 \uparrow}(P-K) G_{\downarrow}(K) \\
& \chi_{\downarrow \uparrow}(P)=\sum_{K} G_{0 \downarrow}(P-K) G_{\uparrow}(K)
\end{aligned}
$$

where $P=\left(i \Omega_{l}, \mathbf{p}\right)$, and $G$ and $G_{0}$ are the full and bare Green's functions respectively. We will discuss $G$ in more detail on a case by case basis. Here $G_{0, \sigma}^{-1}(K)=i \omega_{n}-\xi_{\mathbf{k}, \sigma}$, $\xi_{\mathbf{k}, \sigma}=\epsilon_{\mathbf{k}}-\mu_{\sigma}, \epsilon_{\mathbf{k}}=\hbar^{2} k^{2} / 2 m$ is the kinetic energy of fermions, and $\mu_{\sigma}$ is the fermionic chemical potential for spin $\sigma=\uparrow, \downarrow$. Throughout this paper, we take $\hbar=1, k_{B}=1$, and use the four-vector notation $K \equiv\left(i \omega_{n}, \mathbf{k}\right), P \equiv\left(i \Omega_{l}, \mathbf{q}\right)$, $\sum_{K} \equiv T \sum_{n} \sum_{\mathbf{k}}$, etc, where $\omega_{n}=(2 n+1) \pi T$ and $\Omega_{l}=2 l \pi T$ are the standard odd and even Matsubara frequencies [44] (where $n$ and $l$ are intergers).

For the mean field discussions in Sections [IIII) of this paper we will not be considering general values of $P$ but only zero frequency limits with special values of $\mathbf{p}$ associated with Sarma $(\mathbf{p}=0)$ and $\operatorname{LOFF}(\mathbf{p}=\mathbf{q} \neq 0)$ states. However, when we include the contribution of non-condensed pairs (or pseudogap effects) the general values of $P$ become important. For the Sarma phase we have the BEC condition

$$
t^{-1}(0)=0=U^{-1}+\chi(0)
$$

More generally, for LOFF-like states we have the BEC condition at finite q:

$$
U^{-1}+\chi(0, \mathbf{q})=0
$$

We will discuss, in considerable detail, the nature of the mean field self energy which appears in the full Green's function 
$G_{\sigma}(K)$. Not only does this determine the gap equation but it also leads to the number equations which can be written in terms of Green's functions as $n_{\sigma}=\sum_{K} G_{\sigma}(K)$.

The number and gap equations then provide the underlying basis for the mean field approach. And we will see that the same propagator for non-condensed pairs $[t(P)]$ enters into the beyond-mean-field corrections. It is important to stress that when we refer to "mean field" based approaches in sections $\Pi$ and 【III of this paper, we will not be distinguishing between the order parameter $\Delta_{s c}$ and the excitation gap $\Delta$. Subsequently we will show that this distinction is actually an important one in all but the BCS limit. Specifically, we note that the expressions we present in Sections $\amalg$ and $\amalg$ within our Green's function-based formulation, are more generally valid below $T_{c}$, but for the excitation gap $\Delta$, not for the order parameter.

\section{GAP AND NUMBER EQUATIONS OF SARMA AND ONE PLANE WAVE LOFF PHASES}

\section{A. Mean Field Sarma State}

We now want to study the Sarma or breached pair state. On the fermionic side of resonance, the dispersion relation of one of the two quasi-particle bands ( $E_{k, \uparrow}$ and $E_{k, \downarrow}$, defined below) has two zero crossings, at momenta $k_{1}(\geq 0)$ and $k_{2}$ $\left(>k_{1}\right)$. This is associated with gapless excitations. In a momentum space representation, pairing is confined to $k<k_{1}$ and $k>k_{2}$. This pairing is "breached" by a normal component in the intermediate region $k_{1}<k<k_{2}$. It is this normal component, then, which carries the bulk of the polarization.

If we consider pairing between $\mathbf{k}$ and $-\mathbf{k}$ states, the mean field Hamitonian can be rewritten as

$$
\begin{aligned}
H^{M F}= & \sum_{\mathbf{k}}\left\{\xi_{\mathbf{k}, \uparrow} c_{\mathbf{k}, \uparrow}^{\dagger} c_{\mathbf{k}, \uparrow}+\xi_{\mathbf{k}, \downarrow} c_{-\mathbf{k}, \downarrow}^{\dagger} c_{-\mathbf{k}, \downarrow}\right. \\
& \left.+\Delta c_{-\mathbf{k}, \downarrow}^{\dagger} c_{\mathbf{k}, \uparrow}^{\dagger}+\Delta c_{\mathbf{k}, \uparrow} c_{-\mathbf{k}, \downarrow}\right\},
\end{aligned}
$$

where we have chosen $\Delta^{*}=\Delta$ to be real. Using standard Bogoliubov diagonalization techniques, we readily arrive at the mean field gap equation via the self-consistency condition

$$
\Delta \equiv \sum_{K} U\left\langle c_{k, \uparrow} c_{-k, \downarrow}\right\rangle
$$

which can be written as

$$
\begin{aligned}
0 & =\frac{1}{U}+\sum_{\mathbf{k}}\left[\frac{1-f\left(E_{k \downarrow}\right)-f\left(E_{k \uparrow}\right)}{2 E_{k}}\right] \\
& =\frac{1}{U}+\sum_{\mathbf{k}} \frac{1-2 \bar{f}\left(E_{k}\right)}{2 E_{k}} .
\end{aligned}
$$

Here $\mu=\left(\mu_{\uparrow}+\mu_{\downarrow}\right) / 2$ and $h=\left(\mu_{\uparrow}-\mu_{\downarrow}\right) / 2, E_{\mathbf{k}}=$ $\sqrt{\xi_{\mathbf{k}}^{2}+\Delta^{2}}, E_{k \uparrow}=-h+E_{k}$ and $E_{k \downarrow}=h+E_{k}$, where $\xi_{k}=\epsilon_{k}-\mu$. In addition, we define the average $\bar{f}(x) \equiv$ $[f(x+h)+f(x-h)] / 2$, where $f(x)$ is the Fermi distribution function. The coupling constant $U$ can be replaced in favor of the dimensionless parameter, $1 / k_{F} a$, via the relationship $m /(4 \pi a)=1 / U+\sum_{\mathbf{k}}\left(2 \epsilon_{k}\right)^{-1}$, where $a$ is the two-body $s$-wave scattering length, and $k_{F}$ is the noninteracting Fermi wave vector for the same total number density in the absence of population imbalance. Therefore the gap equation can be rewriten as

$$
-\frac{m}{4 \pi a}=\sum_{\mathbf{k}}\left[\frac{1-2 \bar{f}\left(E_{k}\right)}{2 E_{k}}-\frac{1}{2 \epsilon_{k}}\right] .
$$

This is a familiar gap equation which has appeared many times in the literature. The mean field number equations can be readily deduced

$$
n_{\sigma}=\sum_{\mathbf{k}}\left[f\left(E_{k \sigma}\right) u_{\mathbf{k}}^{2}+f\left(E_{k \bar{\sigma}}\right) v_{\mathbf{k}}^{2}\right]
$$

where $\bar{\sigma}=-\sigma$ and the coherence factors $u_{\mathbf{k}}^{2}, v_{\mathbf{k}}^{2}=(1 \pm$ $\left.\xi_{\mathbf{k}} / E_{\mathbf{k}}\right) / 2$. Equivalently, they can be rewritten as

$$
\begin{aligned}
n & =2 \sum_{\mathbf{k}}\left[v_{\mathbf{k}}^{2}+\frac{\xi_{\mathbf{k}}}{E_{\mathbf{k}}} \bar{f}\left(E_{\mathbf{k}}\right)\right], \\
p n & =\sum_{\mathbf{k}}\left[f\left(E_{\mathbf{k}}-h\right)-f\left(E_{\mathbf{k}}+h\right)\right],
\end{aligned}
$$

where $n=n_{\uparrow}+n_{\downarrow}$ is the total atomic density, $\delta n=n_{\uparrow}-n_{\downarrow}>$ 0 is the number difference and $p=\delta n / n$ is the polarization.

\section{B. T-matrix Approach Below $T_{c}$ : Sarma State}

We next show that the same results can be obtained from a $T$-matrix based approach. This discussion provides the link between the first line of Table I and the previous subsection. The one particle Green's function for particles with spin $\sigma$ is

$$
\begin{aligned}
G_{\sigma}^{-1}(K) & =G_{0 \sigma}^{-1}(K)-\Sigma_{\sigma}(K) \\
& =i \omega_{n}-\xi_{k \sigma}-\Sigma_{\sigma}(K),
\end{aligned}
$$

The self-energy $\Sigma_{\sigma}$ can be shown to be of the BCS-like form

$$
\Sigma_{\sigma}(K)=-\Delta^{2} G_{0 \bar{\sigma}}(-K)=\frac{\Delta^{2}}{i \omega+\xi_{k \bar{\sigma}}} .
$$

We will see later in Section IV how this form for the self energy very naturally arises (below $T_{c}$ ) in a $T$-matrix approach. Thus

$$
G_{\sigma}^{-1}(K)=i \omega-\xi_{k \sigma}-\frac{\Delta^{2}}{i \omega+\xi_{k \bar{\sigma}}} .
$$

Then, using the coherence factors we defined earlier, the Green's functions become

$$
G_{\sigma}(K)=\frac{u_{k}^{2}}{i \omega-E_{k \sigma}}+\frac{v_{k}^{2}}{i \omega+E_{k \bar{\sigma}}} .
$$


Now we are in position to calculate the pair susceptibility at $P=0$ for the Sarma phase based on Eqs. (2) and (3).

$$
\begin{aligned}
\chi(0) & =\chi_{\uparrow \downarrow}(0)=\chi_{\downarrow \uparrow}(0) \\
& =-\sum_{K} \frac{1}{\left(i \omega_{n}-E_{k \downarrow}\right)\left(i \omega_{n}+E_{k \uparrow}\right)} .
\end{aligned}
$$

Substituting this expression into our BEC condition Eq. (4), we obtain the same gap equation (8), after carrying out the Matsubara summation.

In terms of Green's functions, we readily arrive at the number equations: $n_{\sigma}=\sum_{K} G_{\sigma}(K)$, which reduce to the number equations (10) we found earlier.

\section{Mean Field Theory of One Plane Wave LOFF State}

If we now consider condensates in which momentum $\mathbf{k}$ pairs with $-\mathbf{k}+\mathbf{q}$, for, as yet undermined $\mathbf{q}$, the mean field Hamiltonian can be rewritten as

$$
\begin{aligned}
H^{M F}= & \sum_{\mathbf{k}}\left\{\xi_{\mathbf{k}, \uparrow} c_{\mathbf{k}, \uparrow}^{\dagger} c_{\mathbf{k}, \uparrow}+\xi_{\mathbf{k}-\mathbf{q}, \downarrow} c_{-\mathbf{k}+\mathbf{q}, \downarrow}^{\dagger} c_{-\mathbf{k}+\mathbf{q}, \downarrow}\right. \\
& \left.+\Delta c_{-\mathbf{k}+\mathbf{q}, \downarrow}^{\dagger} c_{\mathbf{k}, \uparrow}^{\dagger}+\Delta c_{\mathbf{k}, \uparrow} c_{-\mathbf{k}+\mathbf{q}, \downarrow}\right\}
\end{aligned}
$$

Upon Bogoliubov diagonalization, the self-consistency condition

$$
\Delta \equiv \sum_{K} U\left\langle c_{k, \uparrow} c_{-k+q, \downarrow}\right\rangle
$$

readily leads to the mean-field gap equation

$$
0=\frac{1}{U}+\sum_{\mathbf{k}} \frac{1-f\left(E_{1, \downarrow}\right)-f\left(E_{1, \uparrow}\right)}{2 E_{k q}}
$$

with $E_{1, \uparrow}=E_{k q}-h+\left(\epsilon_{k}-\epsilon_{k-q}\right) / 2, E_{1, \downarrow}=E_{k q}+h-$ $\left(\epsilon_{k}-\epsilon_{k-q}\right) / 2, E_{k q}=\sqrt{\xi_{k q}^{2}+\Delta^{2}}, \xi_{k q}=\left(\xi_{k}+\xi_{k-q}\right) / 2$.

The regularized gap equation is

$$
-\frac{m}{4 \pi a}=\sum_{\mathbf{k}}\left[\frac{1-f\left(E_{1 \uparrow}\right)-f\left(E_{1 \downarrow}\right)}{2 E_{k q}}\right] .
$$

Finally, the mean field number equations for $n_{\sigma}$, are given by

$$
n_{\sigma}=\sum_{\mathbf{k}}\left[f\left(E_{1, \sigma}\right) u_{k q}^{2}+f\left(-E_{1, \bar{\sigma}}\right) v_{k q}^{2}\right] .
$$

Here the coherence factors are $u_{k q}^{2}=\frac{1}{2}\left(1+\frac{\xi_{k q}}{E_{k q}}\right)$ and $v_{k q}^{2}=$ $\frac{1}{2}\left(1-\frac{\xi_{k q}}{E_{k q}}\right)$.

There must be another equation which governs $q$. This can be obtained by minimizing the thermodynamical potential or free energy with respect to q. Equivalently, we will derive $\mathbf{q}$ from the $T$-matrix method described below.

\section{D. $T$-matrix Approach Below $T_{c}$ : One plane wave LOFF state}

We now use the same $T$-matrix based approach (as we did for the Sarma case, and as indicated by Table I) to make contact with the one plane wave LOFF state. We take the self energy of the form

$$
\Sigma_{\sigma}(K)=-\Delta^{2} G_{0 \bar{\sigma}}(-K)=\frac{\Delta^{2}}{i \omega+\xi_{\mathbf{k}-\mathbf{q}, \bar{\sigma}}}
$$

so that

$$
G_{\sigma}^{-1}(K)=i \omega-\xi_{\mathbf{k}, \sigma}-\frac{\Delta^{2}}{i \omega+\xi_{\mathbf{k}-\mathbf{q}, \bar{\sigma}}} .
$$

Then we have

$$
\begin{aligned}
& G_{\uparrow}(K)=\frac{u_{k}^{2}}{i \omega_{n}-E_{1, \uparrow}}+\frac{v_{k}^{2}}{i \omega_{n}+E_{1, \downarrow}} \\
& G_{\downarrow}(K)=\frac{u_{k}^{2}}{i \omega_{n}-E_{2, \downarrow}}+\frac{v_{k}^{2}}{i \omega_{n}+E_{2, \uparrow}}
\end{aligned}
$$

Here $E_{2, \uparrow}=E_{k q}-h-\left(\epsilon_{k}-\epsilon_{k-q}\right) / 2$ and $E_{2, \downarrow}=E_{k q}+h+$ $\left(\epsilon_{k}-\epsilon_{k-q}\right) / 2$. Note if $\mathbf{k} \rightarrow-\mathbf{k}+\mathbf{q}$, then $E_{2, \uparrow} \rightarrow E_{1, \uparrow}$ and $E_{2, \downarrow} \rightarrow E_{1, \downarrow}$. The number equations are $n_{\sigma}=\sum_{K} G_{\sigma}(K)$, which yields the same answer (Eq.21) we found in the mean field approach. For $T \leq T_{c}$, using the BEC condition $U^{-1}+\chi(0, \mathbf{q})=0$ we thus arrive at the gap equation we found earlier in Eq. (19).

We next investigate the pair susceptibility and its extremal value at $Q=(0, \mathbf{q})$. We define the contributions to the pair susceptibility in the presence of population imbalance as in Eqs.(1)-(5). For the one plane wave LOFF state, the BEC condition is that $t(0, \mathbf{p})$ diverges at a non-zero momentum $\mathbf{p}=\mathbf{q}$. Thus, at this momentum, the quantity $\chi(0, \mathbf{p})$ should reach a maximum at $\mathbf{p}=\mathbf{q}$. We determine $\mathbf{q}$ by requiring

$$
\left.\frac{\partial \chi(0, \mathbf{p})}{\partial \mathbf{p}}\right|_{\mathbf{p}=\mathbf{q}}=0
$$

Where $\chi(P)$ is explicitly shown in Eq. A1. This yields

$$
\begin{aligned}
0= & \left.\frac{\partial \chi(0, \mathbf{p})}{\partial \mathbf{p}}\right|_{\mathbf{p}=\mathbf{q}} \\
= & \frac{1}{\Delta^{2}} \sum_{\mathbf{k}}\left\{\frac{\mathbf{q}}{2}\left[\left(1-\frac{\xi_{k q}}{E_{k q}}\right)-\left[f\left(E_{1, \uparrow}\right)+f\left(E_{1, \downarrow}\right)\right] \frac{\xi_{k q}}{E_{k q}}\right]\right. \\
& \left.+\left(\mathbf{k}-\frac{\mathbf{q}}{2}\right)\left[f\left(E_{1, \uparrow}\right)-f\left(E_{1, \downarrow}\right)\right]\right\} .
\end{aligned}
$$

It is important to stress, as we show in Appendix A that this extremal condition on $\chi(P)$ is equivalent to the condition that the net current is identically zero in this situation.

$$
\mathbf{j}=\sum_{K} \mathbf{k}\left[G_{\uparrow}(K)+G_{\downarrow}(K)\right]=0
$$

This key observation shows that the present way of computing q directly from the pair susceptibility is consistent with the 
counterparts in the literature, which are based on the vanishing of the net current in equilibrium.

At the mean field level we, then, have four unknowns $\Delta, \mathbf{q}$, $\mu$ and $h$, and four equations: two number equations, the gap equation, and the condition on the vanishing of the first order derivative.

\section{MEAN FIELD THEORY OF TWO PLANE WAVE LOFF STATE}

The two plane wave LOFF state has not been studied in much detail away from $T_{c}$ and the associated tri-critical point [32]. Here we address this state at more general temperatures, starting with the very natural mean field Hamiltonian in the same spirit as in the previous two cases. The Hamiltonian is given by

$$
\begin{aligned}
H^{M F}= & \sum_{\mathbf{k}}\left\{\xi_{\mathbf{k} \uparrow} c_{\mathbf{k} \uparrow}^{\dagger} c_{\mathbf{k} \uparrow}+\xi_{\mathbf{k} \downarrow} c_{\mathbf{k} \downarrow}^{\dagger} c_{\mathbf{k} \downarrow}\right. \\
& +\frac{1}{\sqrt{2}} \Delta\left(c_{\mathbf{k} \uparrow}^{\dagger} c_{-\mathbf{k}+\mathbf{q} \downarrow}^{\dagger}+c_{\mathbf{k} \downarrow} c_{-\mathbf{k}+\mathbf{q} \uparrow}\right) \\
& \left.+\frac{1}{\sqrt{2}} \Delta\left(c_{\mathbf{k} \uparrow}^{\dagger} c_{-\mathbf{k}-\mathbf{q} \downarrow}^{\dagger}+c_{\mathbf{k} \downarrow} c_{-\mathbf{k}-\mathbf{q} \uparrow}\right)\right\}
\end{aligned}
$$

To solve for the value of the gap we generalize the Bogoliubov diagonalization procedure. If one rewrites the effective Hamiltonian in the basis set $\mathbf{B}_{\uparrow}^{T}=\left(c_{\mathbf{k} \uparrow}, c_{-\mathbf{k}-\mathbf{q} \downarrow}^{\dagger}, c_{-\mathbf{k}+\mathbf{q} \downarrow}^{\dagger}\right)$ and $\mathbf{B}_{\downarrow}^{T}=\left(c_{\mathbf{k} \downarrow}, c_{-\mathbf{k}-\mathbf{q} \uparrow}^{\dagger}, c_{-\mathbf{k}+\mathbf{q} \uparrow}^{\dagger}\right)$, the Hamiltonian can be written in two $3 \times 3$ matrices. Here we show the matrix for $\mathbf{B}_{\uparrow}$ :

$$
\mathbf{H}_{\uparrow}=\left(\begin{array}{ccc}
\xi_{\mathbf{k} \uparrow} & \frac{1}{\sqrt{2}} \Delta & \frac{1}{\sqrt{2}} \Delta \\
\frac{1}{\sqrt{2}} \Delta & -\xi_{\mathbf{k}+\mathbf{q} \downarrow} & 0 \\
\frac{1}{\sqrt{2}} \Delta & 0 & -\xi_{\mathbf{k}-\mathbf{q} \downarrow}
\end{array}\right) .
$$

We can equivalently write the $\uparrow$ component of the $3 \times 3$ matrix in terms of the basis $\mathbf{B}_{\uparrow}^{\prime T}=\left(c_{\mathbf{k}+\mathbf{q} \uparrow}, c_{\mathbf{k}-\mathbf{q} \uparrow}, c_{-\mathbf{k} \downarrow}^{\dagger}\right)$ The mean field gap equation is derived from the averages obtained from a symmetric combination of the two basis sets as

$$
\Delta=\sum_{K} U\left\langle c_{k, \uparrow} c_{-k-q, \downarrow}\right\rangle=\sum_{K} U\left\langle c_{k-q, \uparrow} c_{-k, \downarrow}\right\rangle,
$$

which is equal to

$$
\Delta=\sum_{K} U\left\langle c_{k, \uparrow} c_{-k+q, \downarrow}\right\rangle=\sum_{K} U\left\langle c_{k+q, \uparrow} c_{-k, \downarrow}\right\rangle .
$$

We choose this symmetrized form to write down the gap equation, which reflects the underlying symmetry of the Hamiltonian.

The resulting gap equation can be shown to be of the form

$$
\begin{aligned}
\frac{1}{U}= & \frac{1}{2} \sum_{\mathbf{k}}\left\{\left[f\left(E_{1 \uparrow}\right)+f\left(E_{1 \downarrow}\right)\right] \frac{E_{1}+\mathcal{E}_{k q}}{\left(E_{1}-E_{2}\right)\left(E_{1}-E_{3}\right)}\right. \\
& +\left[f\left(E_{2 \uparrow}\right)+f\left(E_{2 \downarrow}\right)\right] \frac{E_{2}+\mathcal{E}_{k q}}{\left(E_{2}-E_{1}\right)\left(E_{2}-E_{3}\right)} \\
& \left.+\left[f\left(E_{3 \uparrow}\right)+f\left(E_{3 \downarrow}\right)\right] \frac{E_{3}+\mathcal{E}_{k q}}{\left(E_{3}-E_{1}\right)\left(E_{3}-E_{2}\right)}\right\}
\end{aligned}
$$

Here we define $E_{1}, E_{2}$, and $E_{3}$ as the solutions to the cubic equation which is related to the determinant of the $3 \times 3$ eigenvalue equation

$x^{3}+\mathcal{E}_{k q} x^{2}-\left[\tilde{E}_{k q}^{2}+\left(\frac{\mathbf{k} \cdot \mathbf{q}}{m}\right)^{2}\right] x-\mathcal{E}_{k q}\left[\tilde{E}_{k q}^{2}-\left(\frac{\mathbf{k} \cdot \mathbf{q}}{m}\right)^{2}\right]=0$.

where $\mathcal{E}_{k q}=\left(k^{2}+\frac{1}{2} q^{2}\right) / 2 m-\mu$ and $\tilde{E}_{k q}=\sqrt{\mathcal{E}_{k q}^{2}+\Delta^{2}}$. Then $E_{j \uparrow}=E_{j}-\left(q^{2} / 4 m\right)-h$ and $E_{j \downarrow}=E_{j}-\left(q^{2} / 4 m\right)+h$ for $j=1,2,3$.

The mean field number equations are

$$
\begin{aligned}
n_{\sigma}= & \sum_{\mathbf{k}}\left[\eta_{k q}^{2} f\left(E_{1 \sigma}\right)+\gamma_{k q}^{2} f\left(E_{2 \sigma}\right)\right. \\
& \left.+\left(1-\eta_{k q}^{2}-\gamma_{k q}^{2}\right) f\left(E_{3 \sigma}\right)\right]
\end{aligned}
$$

where we define

$$
\begin{aligned}
\eta_{k q}^{2} & =\frac{\left(E_{1}+\mathcal{E}_{k q}\right)^{2}-\left(\frac{\mathbf{k} \cdot \mathbf{q}}{m}\right)^{2}}{\left(E_{2}-E_{1}\right)\left(E_{3}-E_{1}\right)} \\
\gamma_{k q}^{2} & =\frac{\left(E_{2}+\mathcal{E}_{k q}\right)^{2}-\left(\frac{\mathbf{k} \cdot \mathbf{q}}{m}\right)^{2}}{\left(E_{1}-E_{2}\right)\left(E_{3}-E_{2}\right)}
\end{aligned}
$$

\section{A. Green's Function Approach Below $T_{c}$ : Two plane wave LOFF state}

For the counterpart mean field theory, approached from a $T$-matrix scheme, we define the self energy as

$$
\begin{aligned}
\Sigma_{\sigma}(K) & =-\frac{1}{2}\left[\Delta^{2} G_{0 \bar{\sigma}}(-K+Q)+\Delta^{2} G_{0 \bar{\sigma}}(-K-Q)\right] \\
& =\frac{1}{2}\left[\frac{\Delta^{2}}{i \omega_{n}+\xi_{\mathbf{k}-\mathbf{q} \bar{\sigma}}}+\frac{\Delta^{2}}{i \omega_{n}+\xi_{\mathbf{k}+\mathbf{q} \bar{\sigma}}}\right]
\end{aligned}
$$

Here $Q=(0, \mathbf{q})$.

The Green's function is then given by

$$
\begin{aligned}
G_{\sigma}(K) & =\frac{1}{G_{0 \sigma}(K)-\Sigma_{\sigma}(K)} \\
& =\frac{\left(i \omega_{n}+\xi_{\mathbf{k}+\mathbf{q} \bar{\sigma}}\right)\left(i \omega_{n}+\xi_{\mathbf{k}-\mathbf{q} \bar{\sigma}}\right)}{\left(i \omega_{n}-E_{1 \sigma}\right)\left(i \omega_{n}-E_{2 \sigma}\right)\left(i \omega_{n}-E_{3 \sigma}\right)} \\
& =\frac{\eta_{k q}^{2}}{i \omega_{n}-E_{1 \sigma}}+\frac{\gamma_{k q}^{2}}{i \omega_{n}-E_{2 \sigma}}+\frac{1-\eta_{k q}^{2}-\gamma_{k q}^{2}}{i \omega_{n}-E_{3 \sigma}}
\end{aligned}
$$

Again, following Eqs. (1)-(5) we can write out the form of the pair susceptibility. For the two plane wave LOFF state, this equation is presented as Eq. B1 in Appendix B This quantity, in turn, enters the gap equation, given by the BEC condition, $1+U \chi(0, \mathbf{q})=0$. We may write this gap equation in compact form as

$$
\frac{1}{U}=\frac{1}{2} \sum_{K, \sigma} \frac{i \omega_{n}+\xi_{\mathbf{k q} \bar{\sigma}}}{\left(i \omega_{n}-E_{1 \sigma}\right)\left(i \omega_{n}-E_{2 \sigma}\right)\left(i \omega_{n}-E_{3 \sigma}\right)}
$$

This will, in turn, reduce to the gap equation 29 we deduced directly from the $3 \times 3$ matrix analysis. 
Similarly, in our Green's function formalism, we have $n_{\sigma}=\sum_{K} G_{\sigma}(K)$, which reduces to the number equations we found in Eq. (31).

Next we determine the momentum $\mathbf{p}$ that maximizes $\chi(P)$ when $\Omega=0$, i.e., we need to find a solution to $\frac{\partial \chi(0, \mathbf{p})}{\partial \mathbf{p}}=0$. Since the Green's function is symmetric under $\mathbf{k} \rightarrow-\mathbf{k}$, it can be shown that $\mathbf{p}=0$ is a solution, which corresponds to the Sarma phase. Here, however, we are interested in a LOFF-like state $\mathbf{p}= \pm \mathbf{q}$. where both signs contribute in a symmetric fashion. Thus we choose $\mathbf{p}=\mathbf{q}$, and, thereby, arrive at the defining equation for the net momentum of the pairs.

$$
\begin{aligned}
0= & \sum_{K, \sigma}\left(\frac{\mathbf{k}-\mathbf{q}}{m}\right)\left\{\frac{i \omega_{n}+\xi_{\mathbf{k}+\mathbf{q} \bar{\sigma}}}{i \omega_{n}+\xi_{\mathbf{k}-\mathbf{q} \bar{\sigma}}} \frac{1}{\left(i \omega_{n}-E_{1 \sigma}\right)\left(i \omega_{n}-E_{2 \sigma}\right)\left(i \omega_{n}-E_{3 \sigma}\right)}\right\} \\
= & \sum_{\mathbf{k}}\left(\frac{\mathbf{k}-\mathbf{q}}{m}\right)\left\{\frac{2\left(\frac{\mathbf{k} \cdot \mathbf{q}}{m}\right)\left[f\left(\xi_{\mathbf{k}-\mathbf{q} \uparrow}\right)+f\left(\xi_{\mathbf{k}-\mathbf{q} \downarrow}\right)-2\right]}{\left(E_{1}+\mathcal{E}_{k q}-\frac{\mathbf{k} \cdot \mathbf{q}}{m}\right)\left(E_{2}+\mathcal{E}_{k q}-\frac{\mathbf{k} \cdot \mathbf{q}}{m}\right)\left(E_{3}+\mathcal{E}_{k q}-\frac{\mathbf{k} \cdot \mathbf{q}}{m}\right)}\right. \\
& +\frac{f\left(E_{1 \uparrow}\right)+f\left(E_{1 \downarrow}\right)}{\left(E_{1}-E_{2}\right)\left(E_{1}-E_{3}\right)} \frac{E_{1}+\mathcal{E}_{k q}+\frac{\mathbf{k} \cdot \mathbf{q}}{m}}{E_{1}+\mathcal{E}_{k q}-\frac{\mathbf{k} \cdot \mathbf{q}}{m}}+\frac{f\left(E_{2 \uparrow}\right)+f\left(E_{2 \downarrow}\right)}{\left(E_{2}-E_{1}\right)\left(E_{2}-E_{3}\right)} \frac{E_{2}+\mathcal{E}_{k q}+\frac{\mathbf{k} \cdot \mathbf{q}}{m}}{E_{2}+\mathcal{E}_{k q}-\frac{\mathbf{k} \cdot \mathbf{q}}{m}} \\
& \left.+\frac{f\left(E_{3 \uparrow}\right)+f\left(E_{3 \downarrow}\right)}{\left(E_{3}-E_{1}\right)\left(E_{3}-E_{1}\right)} \frac{E_{3}+\mathcal{E}_{k q}+\frac{\mathbf{k} \cdot \mathbf{q}}{m}}{E_{3}+\mathcal{E}_{k q}-\frac{\mathbf{k} \cdot \mathbf{q}}{m}}\right\} .
\end{aligned}
$$

This determines the magnitude $\mathbf{q}$; for definiteness we take the direction of $\mathbf{q}$ as along the $z$-axis.

The case of the single plane wave LOFF state should be contrasted. There we showed that there was an intimate relation between the condition that there be no net equilibrium current and the extremal requirement on $\chi(P)$. For the two plane wave LOFF state the current can be shown to be identically zero. Rather, the only condition one has to determine $q$ is the vanishing of the first derivative of the pair susceptibility. A similar condition was imposed in the original LarkinOvchinnikov (LO) paper [21].

(a)

(b)

(c)

(d)

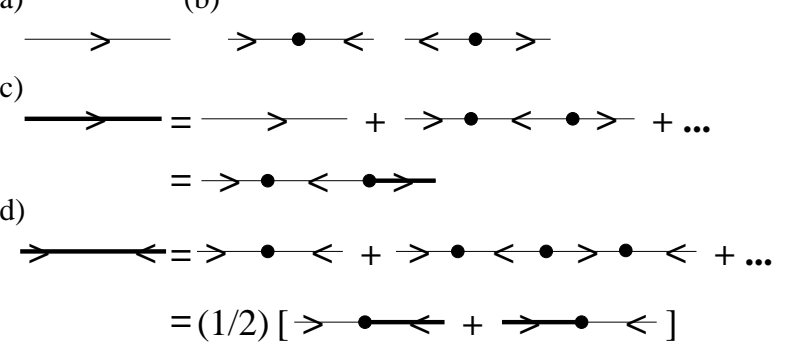

Figure 1: Diagrams for Sarma states or alternatively one plane wave LOFF states including (a) Bare Green's function $G_{0}$. (b) Vertices from pairing. The momenta are $(\mathbf{k} \downarrow,-\mathbf{k} \uparrow)$ and $(\mathbf{k} \uparrow,-\mathbf{k} \downarrow)$, while for single plane-wave LOFF states the momenta are $(\mathbf{k} \downarrow,-\mathbf{k}+\mathbf{q} \uparrow)$ and $(\mathbf{k} \uparrow,-\mathbf{k}+\mathbf{q} \downarrow)$. (c) Green's function $G$. (d) $\left\langle c_{\mathbf{k}} \uparrow c_{-\mathbf{k} \downarrow}\right\rangle$ for Sarma states and $\left\langle c_{\mathbf{k}} \uparrow c_{-\mathbf{k}+\mathbf{q} \downarrow}\right\rangle$ for single plane-wave LOFF states. Here the thin and thick lines represent bare and full Green's functions, respectively.

\section{B. Diagrammatic Interpretation of Green's Function Approaches: Comparison with Larkin-Ovchinnikov}

We now want to compare the present approach for the two plane wave LOFF state with that proposed in the original LO paper [21]. We will do this comparison within a diagrammatic framework. It is useful first to illustrate the diagrammatic scheme by referring to the simpler Sarma and one plane wave LOFF states. These two states have a rather similar diagrammatic formulation. We write these diagrams in a consolidated form in Fig. 11 The first line indicates the bare quantities, that is, the bare Green's function $G_{0}$ in part (a) and the quantity $G_{0, \uparrow}(K) \Delta G_{0, \downarrow}(-K)$ in part (b). This is written for the Sarma state and readily generalized to the one plane wave LOFF phase.

The second line indicates the (diagonal) dressed Green's function, while the third line shows effectively the Gor'kov $F$

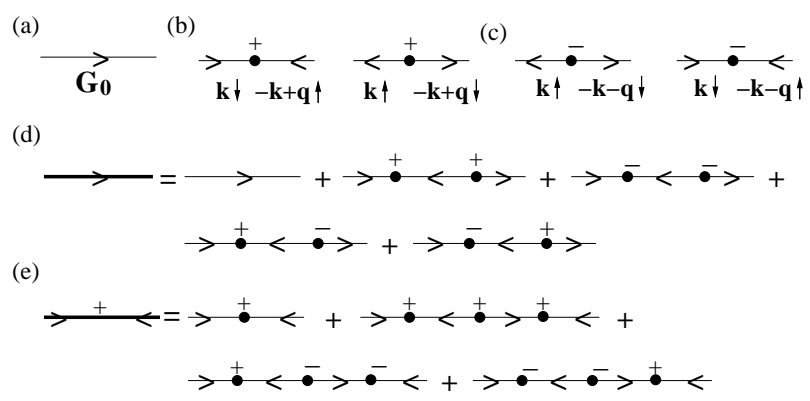

Figure 2: Diagrams representing the original Ovchinnikov-Larkin theory for (a) Non-interacting Green's function $G_{0}$. (b) Vertices from pairing with q. (c) Vertices from pairing with - q. (d) Green's function $G$. (e) $\left\langle c_{\mathbf{k}} \uparrow c_{-\mathbf{k}+\mathbf{q} \downarrow}\right\rangle$. 


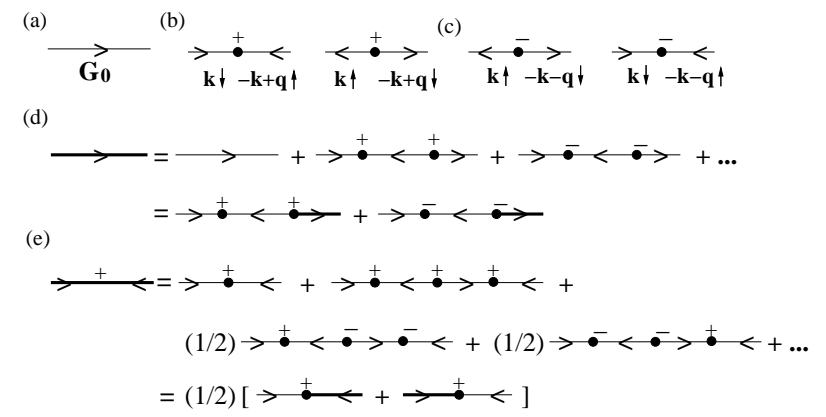

Figure 3: Diagrams associated with the $3 \times 3$ Bogoliubov diagonalization theory for (a) Non-interacting Green's function $G_{0}$. (b) Vertices from pairing with q. (c) Vertices from pairing with - q. (d) Green's function $G$. (e) $\left\langle c_{\mathbf{k}} \uparrow c_{-\mathbf{k}+\mathbf{q} \downarrow}\right\rangle$.

function. It should be noted for Fig. 11 that this latter "anomalous" or off-diagonal Green's function is explicitly seen to depend on a symmetrized sum of the product $G$ and $G_{0}$. It is this combination which we have seen appear in the pair susceptibility $\chi(P)$. Indeed, throughout this paper we have found that $G G_{0}$ is essential for arriving at the standard mean field theo- retic approach. We can, thus, conclude from the last line in the figure that the $F$ function is given by a spin symmetrized combination of $\Delta G G_{0}$.

In Fig. 2] we show the two plane wave LOFF diagrams originally proposed by LO. In order to discuss their implications, we present the diagrams associated with the mean field matrix scheme of the previous section which are plotted in Fig 3 As in Figure 11 one can see from the last line in part e of Fig 3 that the off-diagonal Green's function depends on a symmetrized sum of $G$ and $G_{0}$. One can also see that there are similarities as well as differences in these latter two approaches. In the approach of Section 1 II just as in LO we restrict our calculations to diagrams that do not contain propagators with momentum beyond $\pm \mathbf{k}$ and $\pm \mathbf{k} \pm \mathbf{q}$. The differences are also apparent. In the LO paper, the (diagonal component of the) Green's function diagrams were summed up to second order in $\Delta$. In the gap equation LO dropped diagrams containing propagators with higher momenta and truncated the series at third order in $\Delta$.

If one were to follow the original LO scheme, but sum the entire series, then we will arrive at a slightly modified gap equation. We would find instead the gap is determined by

$$
\begin{aligned}
\frac{1}{U}= & \sum_{K}\left\{\left[G_{0 \uparrow}(-K+Q) G_{\downarrow}(K)+G_{0 \downarrow}(-K+Q) G_{\uparrow}(K)\right]-\frac{1}{2}\left[G_{0 \uparrow}(-K+Q) G_{\downarrow}^{(1)}(K)+G_{0 \downarrow}(-K+Q) G_{\uparrow}^{(1)}(K)\right]\right\} \\
= & \sum_{\mathbf{k}}\left\{\left[f\left(E_{1 \uparrow}\right)+f\left(E_{1 \downarrow}\right)\right] \frac{E_{1}+\mathcal{E}_{k q}}{\left(E_{1}-E_{2}\right)\left(E_{1}-E_{3}\right)}+\left[f\left(E_{2 \uparrow}\right)+f\left(E_{2 \downarrow}\right)\right] \frac{E_{2}+\mathcal{E}_{k q}}{\left(E_{2}-E_{1}\right)\left(E_{2}-E_{3}\right)}\right. \\
& \left.+\left[f\left(E_{3 \uparrow}\right)+f\left(E_{3 \downarrow}\right)\right] \frac{E_{3}+\mathcal{E}_{k q}}{\left(E_{3}-E_{1}\right)\left(E_{3}-E_{2}\right)}\right\}-\sum_{\mathbf{k}} \frac{f\left(E_{1, \uparrow}^{(1)}\right)+f\left(E_{1, \downarrow}^{(1)}\right)-1}{2 E_{k q}^{(1)}}
\end{aligned}
$$

and thus the pair susceptibility derived from this method is

$$
\begin{aligned}
& \chi(P) \\
& \quad=\frac{1}{2} \sum_{K}\left\{\left[G_{0 \uparrow}(P-K) G_{\downarrow}(K)+G_{0 \downarrow}(P-K) G_{\uparrow}(K)\right]\right. \\
& \left.\quad-\left[G_{0 \uparrow}(P-K) G_{\downarrow}^{(1)}(K)+G_{0 \downarrow}(P-K) G_{\uparrow}^{(1)}(K)\right]\right\} .
\end{aligned}
$$

We could similarly use this modified pair susceptibility to arrive at the constraint on the value of $\mathbf{q}$. We discuss these contributions in Appendix B For the above equation we define the Green's function for the single plane-wave LOFF states as $G_{\uparrow}^{(1)}(K)=\left(i \omega_{n}+\xi_{\mathbf{k}-\mathbf{q} \downarrow}\right) /\left(i \omega_{n}-E_{1, \uparrow}^{(1)}\right)\left(i \omega_{n}+E_{1, \downarrow}^{(1)}\right)$ and $G_{\downarrow}^{(1)}(K)=\left(i \omega_{n}+\xi_{\mathbf{k}-\mathbf{q} \uparrow}\right) /\left(i \omega_{n}-E_{2, \downarrow}^{(1)}\right)\left(i \omega_{n}+E_{2, \uparrow}^{(1)}\right)$. The energy spectrum of the single plane-wave LOFF states is $E_{1, \uparrow}^{(1)}=$ $E_{\mathbf{k q}}^{(1)}+\frac{1}{2 m}\left(\mathbf{k} \cdot \mathbf{q}-\frac{q^{2}}{2}\right)-h, E_{1, \downarrow}^{(1)}=E_{\mathbf{k q}}^{(1)}-\frac{1}{2 m}\left(\mathbf{k} \cdot \mathbf{q}-\frac{q^{2}}{2}\right)+h$, $E_{2, \uparrow}^{(1)}=E_{\mathbf{k q}}^{(1)}-\frac{1}{2 m}\left(\mathbf{k} \cdot \mathbf{q}-\frac{q^{2}}{2}\right)-h$, and $E_{2, \downarrow}^{(1)}=E_{\mathbf{k q}}^{(1)}+$ $\frac{1}{2 m}\left(\mathbf{k} \cdot \mathbf{q}-\frac{q^{2}}{2}\right)+h$, where $E_{\mathbf{k q}}^{(1)}=\sqrt{\left(\mathcal{E}_{k q}-\frac{\mathbf{k} \cdot \mathbf{q}}{2 m}\right)^{2}+\Delta_{1}^{2}}$ and we define $\Delta_{1}^{2}=\frac{1}{2} \Delta^{2}$.

In summary, to make contact with the original results of LO, one must subtract a second symmetrized term, which represents pairing with only one momentum. It would thus appear, that relative to $\mathrm{LO}$, there is an overcounting in the summation of the two series from the diagrams shown in Fig 3 and associated with the $3 \times 3$ matrix or mean field representation. At this stage it is difficult to determine which of the two plane wave LOFF representations is the more appropriate. It will be essential in future to study them both numerically in the presence of BCS-BEC crossover effects. 


\section{BEYOND SIMPLE MEAN FIELD THEORY: PAIR FLUCTUATION EFFECTS}

\section{A. Inclusion of the Pseudogap}

For definiteness the equations which appear in this section apply to the Sarma-like phase. We can readily generalize to include the two different LOFF states.

This diagrammatic representation of our $T$-matrix scheme is shown in Figs. 4 and 5 The first of these indicates the propagator for non-condensed pairs which we refer to as $t_{p g}$, and the second of these the total self energy. One can see throughout the combination of one dressed and one bare Green's function, as represented by the thick and thin lines. The self energy consists of two contributions from the non-condensed pairs or pseudogap $(p g)$ and from the condensate $(s c)$. There are, analogously, two contributions in the full $T$-matrix

$$
\begin{aligned}
t & =t_{p g}+t_{s c}, \\
t_{p g}(P) & =\frac{U}{1+U \chi(P)}, \quad P \neq 0, \\
t_{s c}(P) & =-\frac{\Delta_{s c}^{2}}{T} \delta(P),
\end{aligned}
$$

where we write $\Delta_{s c}=-U \sum_{\mathbf{k}}\left\langle c_{-\mathbf{k} \downarrow} c_{\mathbf{k} \uparrow}\right\rangle$. Similarly, we have for the fermion self energy

$$
\Sigma_{\sigma}(K)=\Sigma_{\sigma}^{s c}(K)+\Sigma_{\sigma}^{p g}(K)=\sum_{P} t(P) G_{0, \bar{\sigma}}(P-K) .
$$

We can see at once that

$$
\Sigma_{\sigma}^{s c}(K)=\sum_{P} t_{s c}(P) G_{0, \bar{\sigma}}(P-K)=-G_{0, \bar{\sigma}}(-K) \Delta_{s c}^{2} .
$$

The vanishing of the pair chemical potential implies that

$$
t_{p g}^{-1}(0)=U^{-1}+\chi(0)=0, \quad T \leq T_{c} .
$$

Moreover, a vanishing chemical potential means that $t_{p g}(P)$ is strongly peaked around $P=0$. Thus, we may approximate [45] Eq. (42) to yield

$$
\Sigma_{\sigma}(K) \approx-G_{0, \bar{\sigma}}(-K) \Delta^{2},
$$

where

$$
\Delta^{2}(T) \equiv \Delta_{s c}^{2}(T)+\Delta_{p g}^{2}(T),
$$

Importantly, we are led to identify the quantity $\Delta_{p g}$

$$
\Delta_{p g}^{2} \equiv-\sum_{P \neq 0} t_{p g}(P)
$$

Note that in the normal state (where $\mu_{\text {pair }}$ is non-zero) Eq. 45 is no longer a good approximation.

We now have a closed set of equations for addressing the ordered phase. We can similarly extend this approach to temperatures somewhat above $T_{c}$, by self consistently including a non-zero pair chemical potential. This is a necessary step

$$
t_{p g}=+\square+\square+\square+\square
$$

Figure 4: T-matrix diagrams. Note that there is one dressed and full Green's function. This represents the propagator for the noncondensed pairs.

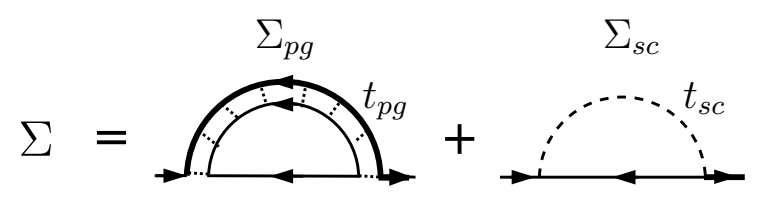

Figure 5: Self energy diagram for the present $T$-matrix scheme, indicating the condensed $\left(\Sigma_{s c}\right)$ and non-condensed $\left(\Sigma_{p g}\right)$ pairs.

in addressing a trap as well [34]. Additionally, the propagator for non-condensed pairs can now be quantified, using the self consistently determined pair susceptibility. At small fourvector $P$, we may expand the inverse of the $T$-matrix, after analytical continuation $\left(i \Omega_{l} \rightarrow \Omega+i 0^{+}\right)$, to obtain

$$
a_{1} \Omega^{2}+Z\left(\Omega-\frac{p^{2}}{2 M^{*}}+\mu_{\text {pair }}+i \Gamma_{P}\right),
$$

where the imaginary part $\Gamma_{P} \rightarrow 0$ rapidly as $p \rightarrow 0$ below $T_{c}$. Because we are interested in the moderate and strong coupling cases, we drop the $a_{1} \Omega^{2}$ term in Eq. (48), and hence

$$
t_{p g}(P)=\frac{Z^{-1}}{\Omega-\Omega_{p}+\mu_{\text {pair }}+i \Gamma_{P}},
$$

where we associate

$$
\Omega_{\mathbf{p}} \equiv \frac{p^{2}}{2 M^{*}}
$$

This establishes a quadratic dispersion and defines the effective pair mass, $M^{*}$.

Finally, one can rewrite Eq. (47) as

$$
\Delta_{p g}^{2}(T)=Z^{-1} \sum_{\mathbf{p}} b\left(\Omega_{p}\right)
$$

where $b(x)$ is the Bose distribution function. Analytical expressions for this mass are possible via a small $\mathbf{p}$ expansion of $\chi$. In this way we find

$$
\chi(P)-\chi(0) \approx Z\left(\Omega-\frac{p^{2}}{2 M^{*}}\right),
$$

The coefficients are

$$
Z=\left.\frac{\partial \chi}{\partial \Omega}\right|_{\Omega=0, \mathbf{p}=0}
$$

and

$$
\frac{1}{2 M^{*}}=-\left.\frac{1}{6 Z} \frac{\partial^{2} \chi}{\partial p^{2}}\right|_{\Omega=0, \mathbf{p}=0}
$$




\section{B. Pair Dispersion in Sarma State and Physical Consequences at Finite $T$}

The theoretical framework in the previous section can now be implemented to address the physics at finite $T$. We do this here only for the Sarma state, thereby expanding on earlier papers [33, 34]. The counterpart results for the two LOFF phases are presented in the two appendices.

We begin by determining the form of the pair dispersion. The pair susceptibility is given by

$$
\begin{aligned}
& \chi(P)=\frac{1}{2}\left[\chi_{\uparrow \downarrow}(P)+\chi_{\downarrow \uparrow}(P)\right] \\
& \quad=\sum_{\mathbf{k}}\left\{u_{k}^{2} \frac{\bar{f}\left(E_{k}\right)+\bar{f}\left(\xi_{p-k}\right)-1}{i \Omega-\xi_{p-k}-E_{k}}+v_{k}^{2} \frac{\bar{f}\left(\xi_{p-k}\right)-\bar{f}\left(E_{k}\right)}{i \Omega-\xi_{p-k}+E_{k}}\right\} .
\end{aligned}
$$

Inserting this form into the $T$-matrix [Eq. (1)] we readily find that the coefficent $Z$ is

$$
\begin{aligned}
Z & =\left.\frac{\partial \chi}{\partial \Omega}\right|_{\Omega=0, \mathbf{p}=0} \\
& =\frac{1}{2 \Delta^{2}}\left[n-2 \sum_{\mathbf{k}} \bar{f}\left(\xi_{k}\right)\right] .
\end{aligned}
$$

Here $n=\sum_{\mathbf{k}, \sigma} n_{\sigma}(k)$ is the total density.

Next we want to calculate $\Omega_{p}$ (defined above) and its derivatives.

$$
\begin{aligned}
\Omega_{p}= & -\left.\frac{1}{Z} t^{-1}\right|_{\Omega=0} \\
= & -\frac{1}{Z}\left\{\frac{m}{4 \pi a}-\sum_{\mathbf{k}}\left[\frac{1}{2 \epsilon_{k}}+u_{k}^{2} \frac{1-\bar{f}\left(E_{k}\right)-\bar{f}\left(\xi_{p-k}\right)}{E_{k}+\xi_{p-k}}\right.\right. \\
& \left.\left.+v_{k}^{2} \frac{\bar{f}\left(\xi_{p-k}\right)-\bar{f}\left(E_{k}\right)}{E_{k}-\xi_{p-k}}\right]\right\}
\end{aligned}
$$

Since $\xi_{k}=k^{2} / 2 m-\mu$, we have $\nabla_{k} \xi_{k}=\mathbf{k} / m$ and $\nabla_{k}^{2} \xi_{k}=$ $3 / m$. Then

$$
\begin{aligned}
\frac{1}{2 M^{*}}= & \left.\frac{1}{6} \frac{\partial^{2} \Omega_{p}}{\partial p^{2}}\right|_{\mathbf{p}=0} \\
= & -\frac{1}{12 m Z \Delta^{2}} \sum_{\mathbf{k}}\left\{\bar{f}\left(\xi_{k}\right)\left[6+\frac{8 \xi_{k}\left(\xi_{k}+\mu\right)}{m \Delta^{2}}\right]\right. \\
& -\bar{f}\left(E_{k \uparrow}\right)\left[\frac{6 \xi_{k}}{E_{k}}+\frac{4\left(\xi_{k}+\mu\right)\left(E_{k}^{2}+\xi_{k}^{2}\right)}{m E_{k} \Delta^{2}}\right] \\
& +4 \bar{f}^{\prime}\left(\xi_{k}\right) \frac{\left(\xi_{k}+\mu\right)}{m}-3\left(1-\frac{\xi_{k}}{E_{k}}\right) \\
& \left.+\frac{2 E_{k}}{m \Delta^{2}}\left(1-\frac{\xi_{k}}{E_{k}}\right)^{2}\left(\xi_{k}+\mu\right)\right\} .
\end{aligned}
$$

Here $\bar{f}^{\prime}(x)$ is the derivative of $\bar{f}(x)$.

With this dispersion, then one can compute the number of non-condensed pairs, $n_{\text {pair }}=Z \Delta_{p g}^{2}=\sum_{\mathbf{p}} b\left(\Omega_{\mathbf{p}}\right)$. We then have

$$
\Delta_{p g}^{2}=\frac{\left(2 M^{*} T\right)^{3 / 2}}{2 \pi^{2} Z} \frac{\sqrt{\pi}}{4} \zeta\left(\frac{3}{2}\right),
$$

where $\zeta(x)$ is the Riemann zeta function. When $T>T_{c}$, $\Delta_{p g}^{2}=Z^{-1} \sum_{\mathbf{p}} b\left(\Omega_{\mathbf{p}}-\mu_{\text {pair }}\right)$.

Physically, these non-condensed pairs, which appear at finite $T$, have been shown to have important physical consequences. In the homogeneous situation, we have found that finite $T$ stabilizes the superfluid state leading to an "intermediate temperature superfluid" [33, 36]. In addition, the presence of a finite excitation gap at $T_{c}\left[\Delta_{p g}\left(T_{c}\right) \neq 0\right]$ makes the behavior of the superfluid transition temperature more complex than its mean field counterpart, $T_{c}^{M F}$. We frequently find a double-valued structure [33], with superfluidity existing only for temperature intermediate between the two $T_{c}$ 's.

In the trapped situation, we have found [34] that these pairs enter explicitly as the mechanism for carrying polarization within the Sarma phase. At low $T$ there is very little polarization carried by the superfluid core; instead, the region of the trap where $\Delta_{s c}=0$, but $\Delta \neq 0$ - which can be called "the mixed normal region"- carries the bulk of the polarization, much as observed experimentally [13].

\section{PHYSICAL PICTURE: FERMIONIC PAIRING IN ANALOGY TO BEC}

We now return to the strong analogies between this BCSbased or Leggett mean field theory [18] and Bose condensation of point bosons, as summarized in Table I.

We have three central equations.

1. The pair chemical potential must vanish at and below $T_{c}$

$$
\mu_{\text {pair }}=0, \quad\left(T \leq T_{c}\right)
$$

Importantly this condition leads to the mean field gap equations derived in Sections $\llbracket$ and 1 These gap equations then provide a specific value for $\Delta(T)$, according to the different phases being contemplated.

2. There must be a conservation of the total number of (composite) "bosons" in the system. For this condition, our central equation is Eq. (46). Here it is understood that the number of "bosons" is effectively represented by the parameter $\Delta^{2}(T)$. In the fermionic limit, this parameter reflects the number of bosons through the energy which is needed to create fermions, and thereby break the bosons apart. Unlike the point boson case, here the "total boson number" is temperature dependent and has to be self-consistently determined. As expected, in the deep BEC regime, where the fermionic excitations are negligible, the pair density is given by $n_{\text {pair }}=Z \Delta^{2} \approx n / 2$.

3. The number of non-condensed pairs is readily computed in terms of the pair dispersion, just as in conventional BEC. For this condition our central equation is Eq. 51.

Then, just as in conventional BEC, the number of condensed bosons (proportional to $\Delta_{s c}^{2}$ ) is determined by the difference between $\Delta^{2}(T)$ and $\Delta_{p g}^{2}(T)$. This, in turn, determines the transition temperature $T_{c}$ as the lowest temperature(s) in the normal state at which noncondensed pairs exhaust the total weight of $\Delta^{2}$ so that $\Delta_{p g}^{2}=\Delta^{2}$. Solving for 
the "transition temperature" in the absence of pseudogap effects [29, 30, 31] leads to the quantity $T_{c}^{M F}$. More precisely, $T_{c}^{M F}$ should be thought of as the temperature at which the excitation gap $\Delta(T)$ vanishes. This provides a reasonable estimate, for the pairing onset temperature $T^{*}$, (when a stable superfluid phase exists). This is distinguished from the transition temperature. We note that $T^{*}$ represents a smooth crossover rather than an abrupt phase transition.

It should be stressed that the dispersion relation for the noncondensed pairs is quadratic. This appears in the Sarma and both one- and two-plane wave LOFF states. For the latter two states, it can be seen to be closely related to the fact that $\chi(0, \mathbf{q})$ must reach a maximum at $\mathbf{q}$. While one will always find a linear dispersion in the collective mode spectrum [41], within the present class of BCS-BEC crossover theories, the restriction to a $T$-matrix scheme means that there is no feedback from the collective modes onto the pair excitation spectrum. In effect, the $T$-matrix approximation does not incorporate pair-pair interactions at a level needed to arrive at this expected linear dispersion in the pair excitation spectrum. Nevertheless, because essentially all theories which address population imbalance build on the simplest BCS-Leggett mean field theory, there is good reason to first address this level of approximation when including finite temperature effects.

\section{SUMMARY}

It should be clear from the previous sections that the zero and finite temperature theories of population imbalanced superfluids can be consolidated into one general theory, based on the quantity which we call the pair susceptibility, $\chi(P)$. This has a very specific form in the class of mean field theories currently applied to address population imbalance. The quantity $\chi(P)$ enters into the propagator for non-condensed pairs Eq. 40 which is just the $T$-matrix, $t(P)$. When noncondensed pairs are in equilibrium with a condensate, they must have zero chemical potential. This, in turn, yields the various gap equations for the Sarma and the one- and twoplane-wave LOFF states, provided one take a special form for the pair susceptibility involving one dressed and one bare Green's function.

Importantly, the same pair propagator characterizes the effective number of non-condensed pairs as seen in Eq. (47). Each of these population imbalanced superfluids has an associated $T \neq 0$ pseudogap contribution, which is summarized for the Sarma case in Section III and for the two LOFF cases in Appendices $\mathrm{A}$ and $\mathrm{B}$ This pseudogap contribution serves to differentiate the order parameter from the gap parameter at all non-zero $T$, and in all cases except strict BCS theory. At a more physical level, in the normal state there is an excitation gap for fermionic excitations associated with "pre-formed" pairs. In the superfluid phase, there is a new form of condensate excitation, not found in BCS theory and associated with excited pair states. As the pairing attraction becomes stronger, it pays to excite pairs of atoms rather than create single fermion excitations which cost an energy gap. These pseudogap effects are an essential component of BCS-BEC crossover theory, and they are necessary in order to smoothly involve from the fermionic statistics of BCS to the bosonic statistics of BEC. Interestingly, they are also widely observed in high temperature superconductors [9, 10].

Thus far, we have applied the present theoretical formalism to the Sarma state both in the homogeneous [33] and trapped [34] configurations. From the point of view of comparing with experiment, our trapped calculations have again underlined the importance of pseudogap effects. We find that the bulk of the polarization is carried in the pseudogap region of the trap: outside the condensate but in the region where both spin states (and thus pairing) are present. Because we find that only modest polarizations are stable, it appears necessary in future to include LOFF-like condensates as well, although their stability must be demonstrated [36]. This observation is also consistent with numerical calculations [25, 29] based on Bogoliubov-de Gennes theory. A key contribution of the present work is that it lays the groundwork for addressing the one and two plane wave LOFF phases, at general temperature.

In summary, this paper has presented a theoretical formalism for the Sarma and one- and two-plane wave LOFF states at zero and finite $T$ as one varies from BCS to BEC, in the presence of an arbitrary population imbalance. Our premise is that the effects of finite $T$, which necessarily must be accomodated in any comparison with experiment, must be compatible with the $T=0$ formalism. The zero temperature formalism we use here reduces to the standard one in the literature [22, 27] for the Sarma and one-plane wave LOFF states. However, our studies of the two-plane wave LOFF state present new results by extending the current literature away from the Landau-Ginzburg regime (near $T_{c}$ ).

\section{Acknowledgments}

This work was supported by NSF PHY-0555325 and NSFMRSEC Grant No. DMR-0213745.

\section{Appendix A: ADDITIONAL RESULTS FOR THE ONE PLANE WAVE LOFF STATE}

The following two appendices are dedicated to presenting additional details on the one- and two-plane-wave LOFF states. The results are presented in appendix form both for clarity and to avoid some of the more technical details in the main text. First, for the one-plane-wave LOFF state, we wish to explore the relationship between the zero current condition applied by Fulde and Ferrell [21] and the extremal condition of $\chi(0, \mathbf{q})$. For the former, we determine momentum $q$ by requiring the total current to be zero. From $\mathbf{j}=\left.\left(\nabla_{\mathbf{x}}-\nabla_{\mathbf{x}^{\prime}}\right)\right|_{x \rightarrow x^{\prime}} \mathbf{G}^{\prime}\left(x, x^{\prime}\right)=\sum_{K}\left[\mathbf{k} G_{\uparrow}(K)+\mathbf{k} G_{\downarrow}(K)\right]$ we get 


$$
\begin{aligned}
\mathbf{j} & =\sum_{\mathbf{k}} \mathbf{k}\left[f\left(E_{1, \uparrow}\right) u_{\mathbf{k}}^{2}+f\left(-E_{1, \downarrow}\right) v_{\mathbf{k}}^{2}\right]-\sum_{\mathbf{k}}(\mathbf{k}-\mathbf{q})\left[f\left(E_{1, \downarrow}\right) u_{\mathbf{k}}^{2}+f\left(-E_{1, \uparrow}\right) v_{\mathbf{k}}^{2}\right] \\
& =\sum_{\mathbf{k}}\left[\frac{\mathbf{q}}{2}\left(1-\frac{\xi_{k q}}{E_{k q}}\right)+\frac{\mathbf{q}}{2}\left[f\left(E_{1, \uparrow}\right)+f\left(E_{1, \downarrow}\right)\right] \frac{\xi_{k q}}{E_{k q}}+\left(\mathbf{k}-\frac{\mathbf{q}}{2}\right)\left[f\left(E_{1, \uparrow}\right)-f\left(E_{1, \downarrow}\right)\right]\right] \\
& =0
\end{aligned}
$$

We can take $\mathbf{q}$ as the $z$ direction, and all the above equations $\quad \chi(P)=\frac{1}{2}\left[\chi_{\uparrow \downarrow}(P)+\chi_{\downarrow \uparrow}(P)\right]$ is need to be integrated over the angular variable $\theta$.

The pair susceptibility, even for the simpler LOFF state is reasonably complex so we present it here for completeness.

$$
\chi(P)=\frac{1}{2} \sum_{\mathbf{k}}\left[u_{k}^{2} \frac{f\left(E_{1, \uparrow}\right)+f\left(E_{2, \downarrow}\right)+f\left(\xi_{p-k, \uparrow}\right)+f\left(\xi_{p-k, \downarrow}\right)-2}{\Omega-\xi_{p-k}-\left(E_{k q}+\left(\epsilon_{k}-\epsilon_{k-q}\right) / 2\right)}+v_{k}^{2} \frac{f\left(\xi_{p-k, \uparrow}\right)+f\left(\xi_{p-k, \downarrow}\right)-f\left(E_{2, \uparrow}\right)-f\left(E_{1, \downarrow}\right)}{\Omega-\xi_{p-k}+\left(E_{k q}-\left(\epsilon_{k}-\epsilon_{k-q}\right) / 2\right)}\right] .
$$

Next, we characterize the pseudogap contributions which enter via $\Delta_{p g}^{2}=Z^{-1} \sum_{\mathbf{p}} b\left(\Omega_{p}\right)$. Here $Z$ and $\frac{1}{2 M^{*}}$ are determined as follows.

$$
\begin{aligned}
Z & =\left.\frac{\partial t^{-1}}{\partial \Omega}\right|_{\Omega=0, \mathbf{p}=\mathbf{q}} \\
& =\frac{1}{2 \Delta^{2}}\left\{n-\sum_{\mathbf{k}}\left[f\left(\xi_{k-q \uparrow}\right)+f\left(\xi_{k-q \downarrow}\right)\right]\right\} .
\end{aligned}
$$

Here $n$ is the total density. Note that the linear derivative vanishes, as was established in Section $\amalg \mathrm{IC}$ Thus we turn next to the quadratic term.

The coefficient of this second order term corresponds to the inverse pair mass, $1 / 2 M^{*}$. This is given by

$$
\begin{aligned}
\frac{1}{2 M^{*}}= & -\left.\frac{1}{6 Z} \frac{\partial^{2} \chi(0, \mathbf{p})}{\partial p^{2}}\right|_{\mathbf{p}=\mathbf{q}} \\
= & -\frac{1}{12 m Z \Delta^{2}} \sum_{\mathbf{k}}\left\{2 \bar{f}\left(\xi_{\mathbf{k}-\mathbf{q}}\right)\left[3+\frac{4 \xi_{k q}(\mathbf{k}-\mathbf{q})^{2}}{m \Delta^{2}}\right]-\left[f\left(E_{1, \uparrow}\right)+f\left(E_{1, \downarrow}\right)\right]\left[\frac{3 \xi_{k q}}{E_{k q}}+\frac{2\left[(\mathbf{k}-\mathbf{q})^{2}+q^{2}\right]\left(E_{k q}^{2}+\xi_{k q}^{2}\right)}{m E_{k q} \Delta^{2}}\right]\right. \\
& +\frac{2\left[f\left(E_{1, \uparrow}\right)-f\left(E_{1, \downarrow}\right)\right]}{m \Delta^{2}}\left[(\mathbf{k}-\mathbf{q})^{2}-q^{2}\right] \xi_{k q}+\frac{8}{m} \bar{f}^{\prime}\left(\xi_{\mathbf{k}-\mathbf{q}}\right)(\mathbf{k}-\mathbf{q})^{2}-3\left(1-\frac{\xi_{k q}}{E_{k q}}\right) \\
& \left.+\frac{2 E_{k q}}{m \Delta^{2}}\left(1-\frac{\xi_{k q}}{E_{k q}}\right)^{2}(\mathbf{k}-\mathbf{q})^{2}\right\}
\end{aligned}
$$

\section{Appendix B: ADDITIONAL RESULTS FOR THE TWO PLANE WAVE LOFF STATE}

We begin with the results that relate to the simplest mean field-based scheme discussed in Section III A We then pro- ceed to the slightly different scheme based on a resummation of the LO diagrams.

The pair susceptibility is given by 


$$
\begin{aligned}
\chi(P) & =\frac{1}{2} \sum_{K}\left[G_{0 \uparrow}(P-K) G_{\downarrow}(K)+G_{0 \downarrow}(P-K) G_{\uparrow}(K)\right] \\
& =\frac{1}{2} T \sum_{n} \sum_{\mathbf{k}, \sigma} \frac{1}{i \Omega-i \omega_{n}-\xi_{\mathbf{p}-\mathbf{k} \sigma}} \frac{\left(i \omega_{n}+\xi_{\mathbf{k}+\mathbf{q} \sigma}\right)\left(i \omega_{n}+\xi_{\mathbf{k}-\mathbf{q} \sigma}\right)}{\left(i \omega_{n}-E_{1 \bar{\sigma}}\right)\left(i \omega_{n}-E_{2 \bar{\sigma}}\right)\left(i \omega_{n}-E_{3 \bar{\sigma}}\right)}
\end{aligned}
$$

From the pair susceptibility one may obtain the dispersion relation for the non-condensed pairs. This is important for introducing pseudogap effects and treating $T \neq 0$.

The explicit forms of the coefficients $Z$ and $1 / 2 M^{*}$ are

$$
\begin{aligned}
Z= & -\frac{1}{2} \sum_{\mathbf{k}}\left\{\frac{\frac{2}{m} \mathbf{k} \cdot \mathbf{q}\left[f\left(\xi_{\mathbf{k}-\mathbf{q} \uparrow}\right)+f\left(\xi_{\mathbf{k}-\mathbf{q} \downarrow}\right)-2\right]}{\left(E_{1}+\mathcal{E}_{k q}-\frac{\mathbf{k} \cdot \mathbf{q}}{m}\right)\left(E_{2}+\mathcal{E}_{k q}-\frac{\mathbf{k} \cdot \mathbf{q}}{m}\right)\left(E_{3}+\mathcal{E}_{k q}-\frac{\mathbf{k} \cdot \mathbf{q}}{m}\right)}+\frac{f\left(E_{1 \uparrow}\right)+f\left(E_{1 \downarrow}\right)}{\left(E_{1}-E_{2}\right)\left(E_{1}-E_{3}\right)} \frac{E_{1}+\mathcal{E}_{k q}+\frac{\mathbf{k} \cdot \mathbf{q}}{m}}{E_{1}+\mathcal{E}_{k q}-\frac{\mathbf{k} \cdot \mathbf{q}}{m}}\right. \\
& \left.+\frac{f\left(E_{2 \uparrow}\right)+f\left(E_{2 \downarrow}\right)}{\left(E_{2}-E_{1}\right)\left(E_{2}-E_{3}\right)} \frac{E_{2}+\mathcal{E}_{k q}+\frac{\mathbf{k} \cdot \mathbf{q}}{m}}{E_{2}+\mathcal{E}_{k q}-\frac{\mathbf{k} \cdot \mathbf{q}}{m}}+\frac{f\left(E_{3 \uparrow}\right)+f\left(E_{3 \downarrow}\right)}{\left(E_{3}-E_{1}\right)\left(E_{3}-E_{1}\right)} \frac{E_{3}+\mathcal{E}_{k q}+\frac{\mathbf{k} \cdot \mathbf{q}}{m}}{E_{3}+\mathcal{E}_{k q}-\frac{\mathbf{k} \cdot \mathbf{q}}{m}}\right\}
\end{aligned}
$$

and

$$
\begin{aligned}
\frac{1}{2 M^{*}}= & \frac{1}{24 m Z} \sum_{\mathbf{k}}\left\{\frac { f ( \xi _ { \mathbf { k } - \mathbf { q } \uparrow } ) + f ( \xi _ { \mathbf { k } - \mathbf { q } \downarrow } ) - 2 } { ( E _ { 1 } + \mathcal { E } _ { k q } - \frac { \mathbf { k } \cdot \mathbf { q } } { m } ) ( E _ { 2 } + \mathcal { E } _ { k q } - \frac { \mathbf { k } \cdot \mathbf { q } } { m } ) ( E _ { 3 } + \mathcal { E } _ { k q } - \frac { \mathbf { k } \cdot \mathbf { q } } { m } ) } \left[-\frac{12}{m}(\mathbf{k} \cdot \mathbf{q})+\frac{4}{m}\left(\xi_{\mathbf{k}-\mathbf{q}}+\mu\right)\right.\right. \\
& +\frac{8}{m^{2}}(\mathbf{k} \cdot \mathbf{q})\left(\xi_{\mathbf{k}-\mathbf{q}}+\mu\right)\left(\frac{1}{E_{1}+\mathcal{E}_{k q}-\frac{\mathbf{k} \cdot \mathbf{q}}{m}}+\frac{1}{E_{2}+\mathcal{E}_{k q}-\frac{\mathbf{k} \cdot \mathbf{q}}{m}}+\frac{1}{\left.\left.E_{3}+\mathcal{E}_{k q}-\frac{\mathbf{k} \cdot \mathbf{q}}{m}\right)\right]}\right. \\
& -\frac{\frac{8}{m^{2}}(\mathbf{k} \cdot \mathbf{q})\left(\xi_{\mathbf{k}-\mathbf{q}}+\mu\right)\left[f^{\prime}\left(\xi_{\mathbf{k}-\mathbf{q} \uparrow}\right)+f^{\prime}\left(\xi_{\mathbf{k}-\mathbf{q} \downarrow}\right)\right]}{\left(E_{1}+\mathcal{E}_{k q}-\frac{\mathbf{k} \cdot \mathbf{q}}{m}\right)\left(E_{2}+\mathcal{E}_{k q}-\frac{\mathbf{k} \cdot \mathbf{q}}{m}\right)\left(E_{3}+\mathcal{E}_{k q}-\frac{\mathbf{k} \cdot \mathbf{q}}{m}\right)} \\
& +\frac{f\left(E_{1 \uparrow}\right)+f\left(E_{1 \downarrow}\right)}{\left(E_{1}-E_{2}\right)\left(E_{1}-E_{3}\right)}\left[\frac{\frac{4}{m}\left(\xi_{\mathbf{k}-\mathbf{q}}+\mu\right)\left(E_{1}+\mathcal{E}_{k q}+\frac{\mathbf{k} \cdot \mathbf{q}}{m}\right)}{\left(E_{1}+\mathcal{E}_{k q}-\frac{\mathbf{k} \cdot \mathbf{q}}{m}\right)^{2}}-6 \frac{E_{1}+\mathcal{E}_{k q}+\frac{\mathbf{k} \cdot \mathbf{q}}{m}}{E_{1}+\mathcal{E}_{k q}-\frac{\mathbf{k} \cdot \mathbf{q}}{m}}\right] \\
& +\frac{f\left(E_{2 \uparrow}\right)+f\left(E_{2 \downarrow}\right)}{\left(E_{2}-E_{1}\right)\left(E_{2}-E_{3}\right)}\left[\frac{\frac{4}{m}\left(\xi_{\mathbf{k}-\mathbf{q}}+\mu\right)\left(E_{2}+\mathcal{E}_{k q}+\frac{\mathbf{k} \cdot \mathbf{q}}{m}\right)}{\left(E_{2}+\mathcal{E}_{k q}-\frac{\mathbf{k} \cdot \mathbf{q}}{m}\right)^{2}}-6 \frac{E_{2}+\mathcal{E}_{k q}+\frac{\mathbf{k} \cdot \mathbf{q}}{m}}{E_{2}+\mathcal{E}_{k q}-\frac{\mathbf{k} \cdot \mathbf{q}}{m}}\right] \\
& \left.+\frac{f\left(E_{2 \uparrow}\right)+f\left(E_{2 \downarrow}\right)}{\left(E_{2}-E_{1}\right)\left(E_{2}-E_{3}\right)}\left[\frac{\frac{4}{m}\left(\xi_{\mathbf{k}-\mathbf{q}}+\mu\right)\left(E_{3}+\mathcal{E}_{k q}+\frac{\mathbf{k} \cdot \mathbf{q}}{m}\right)}{\left(E_{3}+\mathcal{E}_{k q}-\frac{\mathbf{k} \cdot \mathbf{q}}{m}\right)^{2}}-6 \frac{E_{3}+\mathcal{E}_{k q}+\frac{\mathbf{k} \cdot \mathbf{q}}{m}}{E_{3}+\mathcal{E}_{k q}-\frac{\mathbf{k} \cdot \mathbf{q}}{m}}\right]\right\}
\end{aligned}
$$

For the LO-based pair susceptibility we have

$$
\begin{aligned}
\chi^{L O}(P)= & \sum_{K}\left[G_{0 \uparrow}(P-K) G_{\downarrow}(K)+G_{0 \downarrow}(P-K) G_{\uparrow}(K)\right]-\frac{1}{2} \sum_{K}\left[G_{0 \uparrow}(P-K) G_{\downarrow}^{(1)}(K)+G_{0 \downarrow}(P-K) G_{\uparrow}^{(1)}(K)\right] \\
= & T \sum_{n} \sum_{\mathbf{k}, \sigma}\left\{\frac{1}{i \Omega_{l}-i \omega_{n}-\xi_{\mathbf{p}-\mathbf{k} \sigma}} \frac{\left(i \omega_{n}+\xi_{\mathbf{k}+\mathbf{q} \sigma}\right)\left(i \omega_{n}+\xi_{\mathbf{k}-\mathbf{q} \sigma}\right)}{\left(i \omega_{n}-E_{1 \bar{\sigma}}\right)\left(i \omega_{n}-E_{2 \bar{\sigma}}\right)\left(i \omega_{n}-E_{3 \bar{\sigma}}\right)}\right\} \\
& -\frac{1}{2} T \sum_{n} \sum_{\mathbf{k}}\left\{\frac{1}{i \Omega_{l}-i \omega_{n}-\xi_{\mathbf{p}-\mathbf{k} \uparrow}} \frac{i \omega_{n}+\xi_{\mathbf{k}-\mathbf{q} \uparrow}}{\left(i \omega_{n}-E_{2, \downarrow}^{(1)}\right)\left(i \omega_{n}+E_{2, \uparrow}^{(1)}\right)}\right. \\
& \left.+\frac{1}{i \Omega_{l}-i \omega_{n}-\xi_{\mathbf{p}-\mathbf{k} \downarrow}} \frac{i \omega_{n}+\xi_{\mathbf{k}-\mathbf{q} \downarrow}}{\left(i \omega_{n}-E_{1, \uparrow}^{(1)}\right)\left(i \omega_{n}+E_{1, \downarrow}^{(1)}\right)}\right\}
\end{aligned}
$$


The momentum $\mathbf{q}$ is determined by minimizing $\chi(0, \mathbf{p})$, i.e., $\frac{\partial \chi(0, \mathbf{p})}{\partial \mathbf{p}}=0$ at $\mathbf{p}=\mathbf{q}$. This gives the momentum equation

$$
\begin{aligned}
0= & T \sum_{n} \sum_{\mathbf{k}, \sigma}\left(\frac{\mathbf{k}-\mathbf{q}}{m}\right) \frac{i \omega_{n}+\xi_{\mathbf{k}+\mathbf{q} \sigma}}{i \omega_{n}+\xi_{\mathbf{k}-\mathbf{q} \sigma}} \frac{1}{\left(i \omega_{n}-E_{1 \bar{\sigma}}\right)\left(i \omega_{n}-E_{2 \bar{\sigma}}\right)\left(i \omega_{n}-E_{3 \bar{\sigma}}\right)} \\
& -\frac{1}{2} T \sum_{n} \sum_{\mathbf{k}}\left(\frac{\mathbf{k}-\mathbf{q}}{m}\right)\left[\frac{1}{i \omega_{n}+\xi_{\mathbf{k}-\mathbf{q} \uparrow}} \frac{1}{\left(i \omega_{n}-E_{2, \downarrow}^{(1)}\right)\left(i \omega_{n}+E_{2, \uparrow}^{(1)}\right)}+\frac{1}{i \omega_{n}+\xi_{\mathbf{k}-\mathbf{q} \downarrow}} \frac{1}{\left(i \omega_{n}-E_{1, \uparrow}^{(1)}\right)\left(i \omega_{n}+E_{1, \downarrow}^{(1)}\right)}\right] \\
= & \sum_{\mathbf{k}}\left(\frac{\mathbf{k}-\mathbf{q}}{m}\right)\left\{\frac{\frac{2}{m}(\mathbf{k} \cdot \mathbf{q})\left[f\left(\xi_{\mathbf{k}-\mathbf{q} \uparrow}\right)+f\left(\xi_{\mathbf{k}-\mathbf{q} \downarrow}\right)-2\right]}{\left(E_{1}+\mathcal{E}_{k q}-\frac{\mathbf{k} \cdot \mathbf{q}}{m}\right)\left(E_{2}+\mathcal{E}_{k q}-\frac{\mathbf{k} \cdot \mathbf{q}}{m}\right)\left(E_{3}+\mathcal{E}_{k q}-\frac{\mathbf{k} \cdot \mathbf{q}}{m}\right)}\right. \\
& +\frac{f\left(E_{1 \uparrow}\right)+f\left(E_{1 \downarrow}\right)}{\left(E_{1}-E_{2}\right)\left(E_{1}-E_{3}\right)} \frac{E_{1}+\mathcal{E}_{k q}+\frac{\mathbf{k} \cdot \mathbf{q}}{m}}{E_{1}+\mathcal{E}_{k q}-\frac{\mathbf{k} \cdot \mathbf{q}}{m}}+\frac{f\left(E_{2 \uparrow}\right)+f\left(E_{2 \downarrow}\right)}{\left(E_{2}-E_{1}\right)\left(E_{2}-E_{3}\right)} \frac{E_{2}+\mathcal{E}_{k q}+\frac{\mathbf{k} \cdot \mathbf{q}}{m}}{E_{2}+\mathcal{E}_{k q}-\frac{\mathbf{k} \cdot \mathbf{q}}{m}} \\
& \left.+\frac{f\left(E_{3 \uparrow}\right)+f\left(E_{3 \downarrow}\right)}{\left(E_{3}-E_{1}\right)\left(E_{3}-E_{1}\right)} \frac{E_{3}+\mathcal{E}_{k q}+\frac{\mathbf{k} \cdot \mathbf{q}}{m}}{E_{3}+\mathcal{E}_{k q}-\frac{\mathbf{k} \cdot \mathbf{q}}{m}}\right\} \\
& -\frac{1}{2 \Delta^{2}} \sum_{\mathbf{k}}\left\{\frac{\mathbf{q}}{2}\left(1-\frac{\xi_{\mathbf{k q}}^{(1)}}{E_{k q}^{(1)}}\right)+\frac{\mathbf{q}}{2} \frac{\xi_{\mathbf{k q}}^{(1)}}{E_{k q}^{(1)}}\left[f\left(E_{1, \uparrow}^{(1)}\right)+f\left(E_{1, \downarrow}^{(1)}\right)\right]+\left(\mathbf{k}-\frac{\mathbf{q}}{2}\right)\left[f\left(E_{1, \uparrow}^{(1)}\right)-f\left(E_{1, \downarrow}^{(1)}\right)\right]\right\} .
\end{aligned}
$$

The coefficients in the pseudogap dispersion are

$$
\begin{aligned}
Z= & -\sum_{\mathbf{k}}\left\{\frac{\frac{2}{m}(\mathbf{k} \cdot \mathbf{q})\left[f\left(\xi_{\mathbf{k}-\mathbf{q} \uparrow}\right)+f\left(\xi_{\mathbf{k}-\mathbf{q} \downarrow}\right)-2\right]}{\left(E_{1}+\mathcal{E}_{k q}-\frac{\mathbf{k} \cdot \mathbf{q}}{m}\right)\left(E_{2}+\mathcal{E}_{k q}-\frac{\mathbf{k} \cdot \mathbf{q}}{m}\right)\left(E_{3}+\mathcal{E}_{k q}-\frac{\mathbf{k} \cdot \mathbf{q}}{m}\right)}+\frac{f\left(E_{1 \uparrow}\right)+f\left(E_{1 \downarrow}\right)}{\left(E_{1}-E_{2}\right)\left(E_{1}-E_{3}\right)} \frac{E_{1}+\mathcal{E}_{k q}+\frac{\mathbf{k} \cdot \mathbf{q}}{m}}{E_{1}+\mathcal{E}_{k q}-\frac{\mathbf{k} \cdot \mathbf{q}}{m}}\right. \\
& +\frac{f\left(E_{2 \uparrow}\right)+f\left(E_{2 \downarrow}\right)}{\left(E_{2}-E_{1}\right)\left(E_{2}-E_{3}\right)} \frac{E_{2}+\mathcal{E}_{k q}+\frac{\mathbf{k} \cdot \mathbf{q}}{m}}{E_{2}+\mathcal{E}_{k q}-\frac{\mathbf{k} \cdot \mathbf{q}}{m}}+\frac{f\left(E_{3 \uparrow}\right)+f\left(E_{3 \downarrow}\right)}{\left(E_{3}-E_{1}\right)\left(E_{3}-E_{1}\right)} \frac{E_{3}+\mathcal{E}_{k q}+\frac{\mathbf{k} \cdot \mathbf{q}}{m}}{E_{3}+\mathcal{E}_{k q}-\frac{\mathbf{k} \cdot \mathbf{q}}{m}} \\
& \left.+\frac{1}{2 \Delta^{2}}\left[\left[1-f\left(\xi_{\mathbf{k}-\mathbf{q} \uparrow}\right)-f\left(\xi_{\mathbf{k}-\mathbf{q} \downarrow}\right)\right]-\frac{\xi_{\mathbf{k} \cdot \mathbf{q}}^{(1)}}{E_{k q}^{(1)}}\left[1-f\left(E_{1, \uparrow}^{(1)}\right)-f\left(E_{1, \downarrow}^{(1)}\right)\right]\right]\right\}
\end{aligned}
$$

and

$$
\begin{aligned}
& \frac{1}{2 M^{*}}=\frac{1}{12 m Z} \sum_{\mathbf{k}}\left\{\frac { f ( \xi _ { \mathbf { k } - \mathbf { q } \uparrow } ) + f ( \xi _ { \mathbf { k } - \mathbf { q } \downarrow } ) - 2 } { ( E _ { 1 } + \mathcal { E } _ { k q } - \frac { \mathbf { k } \cdot \mathbf { q } } { m } ) ( E _ { 2 } + \mathcal { E } _ { k q } - \frac { \mathbf { k } \cdot \mathbf { q } } { m } ) ( E _ { 3 } + \mathcal { E } _ { k q } - \frac { \mathbf { k } \cdot \mathbf { q } } { m } ) } \left[-\frac{12}{m}(\mathbf{k} \cdot \mathbf{q})+\frac{4}{m}\left(\xi_{\mathbf{k}-\mathbf{q}}+\mu\right)\right.\right. \\
& \left.+\frac{8}{m^{2}}(\mathbf{k} \cdot \mathbf{q})\left(\xi_{\mathbf{k}-\mathbf{q}}+\mu\right)\left(\frac{1}{E_{1}+\mathcal{E}_{k q}-\frac{\mathbf{k} \cdot \mathbf{q}}{m}}+\frac{1}{E_{2}+\mathcal{E}_{k q}-\frac{\mathbf{k} \cdot \mathbf{q}}{m}}+\frac{1}{E_{3}+\mathcal{E}_{k q}-\frac{\mathbf{k} \cdot \mathbf{q}}{m}}\right)\right] \\
& -\frac{\frac{8}{m^{2}}(\mathbf{k} \cdot \mathbf{q})\left(\xi_{\mathbf{k}-\mathbf{q}}+\mu\right)\left[f^{\prime}\left(\xi_{\mathbf{k}-\mathbf{q} \uparrow}\right)+f^{\prime}\left(\xi_{\mathbf{k}-\mathbf{q} \downarrow}\right)\right]}{\left(E_{1}+\mathcal{E}_{k q}-\frac{\mathbf{k} \cdot \mathbf{q}}{m}\right)\left(E_{2}+\mathcal{E}_{k q}-\frac{\mathbf{k} \cdot \mathbf{q}}{m}\right)\left(E_{3}+\mathcal{E}_{k q}-\frac{\mathbf{k} \cdot \mathbf{q}}{m}\right)} \\
& +\frac{f\left(E_{1 \uparrow}\right)+f\left(E_{1 \downarrow}\right)}{\left(E_{1}-E_{2}\right)\left(E_{1}-E_{3}\right)}\left[\frac{\frac{4}{m}\left(\xi_{\mathbf{k}-\mathbf{q}}+\mu\right)\left(E_{1}+\mathcal{E}_{k q}+\frac{\mathbf{k} \cdot \mathbf{q}}{m}\right)}{\left(E_{1}+\mathcal{E}_{k q}-\frac{\mathbf{k} \cdot \mathbf{q}}{m}\right)^{2}}-6 \frac{E_{1}+\mathcal{E}_{k q}+\frac{\mathbf{k} \cdot \mathbf{q}}{m}}{E_{1}+\mathcal{E}_{k q}-\frac{\mathbf{k} \cdot \mathbf{q}}{m}}\right] \\
& +\frac{f\left(E_{2 \uparrow}\right)+f\left(E_{2 \downarrow}\right)}{\left(E_{2}-E_{1}\right)\left(E_{2}-E_{3}\right)}\left[\frac{\frac{4}{m}\left(\xi_{\mathbf{k}-\mathbf{q}}+\mu\right)\left(E_{2}+\mathcal{E}_{k q}+\frac{\mathbf{k} \cdot \mathbf{q}}{m}\right)}{\left(E_{2}+\mathcal{E}_{k q}-\frac{\mathbf{k} \cdot \mathbf{q}}{m}\right)^{2}}-6 \frac{E_{2}+\mathcal{E}_{k q}+\frac{\mathbf{k} \cdot \mathbf{q}}{m}}{E_{2}+\mathcal{E}_{k q}-\frac{\mathbf{k} \cdot \mathbf{q}}{m}}\right] \\
& \left.+\frac{f\left(E_{2 \uparrow}\right)+f\left(E_{2 \downarrow}\right)}{\left(E_{2}-E_{1}\right)\left(E_{2}-E_{3}\right)}\left[\frac{\frac{4}{m}\left(\xi_{\mathbf{k}-\mathbf{q}}+\mu\right)\left(E_{3}+\mathcal{E}_{k q}+\frac{\mathbf{k} \cdot \mathbf{q}}{m}\right)}{\left(E_{3}+\mathcal{E}_{k q}-\frac{\mathbf{k} \cdot \mathbf{q}}{m}\right)^{2}}-6 \frac{E_{3}+\mathcal{E}_{k q}+\frac{\mathbf{k} \cdot \mathbf{q}}{m}}{E_{3}+\mathcal{E}_{k q}-\frac{\mathbf{k} \cdot \mathbf{q}}{m}}\right]\right\} \\
& -\frac{1}{12 m Z \Delta_{1}^{2}} \sum_{\mathbf{k}}\left\{\left[f\left(\xi_{\mathbf{k}-\mathbf{q} \uparrow}\right)+f\left(\xi_{\mathbf{k}-\mathbf{q} \downarrow}\right)\right]\left[3+\frac{4 \xi_{\mathbf{k q}}^{(1)}(\mathbf{k}-\mathbf{q})^{2}}{m \Delta_{1}^{2}}\right]\right. \\
& -\left[f\left(E_{1, \uparrow}^{(1)}\right)+f\left(E_{1, \downarrow}^{(1)}\right)\right]\left[\frac{3 \xi_{\mathbf{k q}}^{(1)}}{E_{k q}^{(1)}}+\frac{2\left[(\mathbf{k}-\mathbf{q})^{2}+q^{2}\right]\left(E_{k q}^{(1)^{2}}+\xi_{\mathbf{k q}}^{(1)^{2}}\right)}{m E_{k q}^{(1)} \Delta_{1}^{2}}\right]+\frac{2\left[f\left(E_{1, \uparrow}^{(1)}\right)-f\left(E_{1, \downarrow}^{(1)}\right)\right]}{m \Delta_{1}^{2}}\left[(\mathbf{k}-\mathbf{q})^{2}-q^{2}\right] \xi_{\mathbf{k q}}^{(1)} \\
& \left.+\frac{2}{m}\left[f^{\prime}\left(\xi_{\mathbf{k}-\mathbf{q} \uparrow}\right)+f^{\prime}\left(\xi_{\mathbf{k}-\mathbf{q} \downarrow}\right)\right](\mathbf{k}-\mathbf{q})^{2}-3\left(1-\frac{\xi_{\mathbf{k q}}^{(1)}}{E_{k q}^{(1)}}\right)+\frac{2 E_{k q}^{(1)}}{m \Delta_{1}^{2}}\left(1-\frac{\xi_{\mathbf{k q}}^{(1)}}{E_{k q}^{(1)}}\right)^{2}(\mathbf{k}-\mathbf{q})^{2}\right\} .
\end{aligned}
$$


Here we define $u_{k q}^{(1)^{2}}=\frac{1}{2}\left(1+\frac{\xi_{\mathbf{k q}}^{(1)}}{E_{k q}^{(1)}}\right)$ and $v_{k q}^{(1)^{2}}=\frac{1}{2}\left(1-\frac{\xi_{\mathbf{k q}}^{(1)}}{E_{k q}^{(1)}}\right)$, where $\xi_{\mathbf{k q}}^{(1)}=\mathcal{E}_{k q}-\mathbf{k} \cdot \mathbf{q} / 2 m$.

[1] M. Greiner, C. A. Regal, and D. S. Jin, Nature 426, 537 (2003).

[2] S. Jochim et al., Science 302, 2101 (2003).

[3] C. A. Regal, M. Greiner, and D. S. Jin, Phys. Rev. Lett. 92, 040403 (2004).

[4] M. W. Zwierlein, C. A. Stan, C. H. Schunck, S. M. F. Raupach, A. J. Kerman, and W. Ketterle, Phys. Rev. Lett. 92, 120403 (2004).

[5] M. W. Zwierlein, J. R. Abo-Shaeer, A. Schirotzek, and W. Ketterle, Nature 435, 170404 (2005).

[6] J. Kinast, S. L. Hemmer, M. E. Gehm, A. Turlapov, and J. E. Thomas, Phys. Rev. Lett. 92, 150402 (2004).

[7] M. Bartenstein, A. Altmeyer, S. Riedl, S. Jochim, C. Chin, J. H. Denschlag, and R. Grimm, Phys. Rev. Lett. 92, 203201 (2004).

[8] J. Kinast, A. Turlapov, J. E. Thomas, Q. J. Chen, J. Stajic, and K. Levin, Science 307, 1296 (2005), published online 27 January 2005; doi:10.1126/science. 1109220

[9] Q. J. Chen, J. Stajic, S. N. Tan, and K. Levin, Phys. Rep. 412, 1 (2005).

[10] Q. J. Chen, J. Stajic, and K. Levin, Low Temp. Phys. 32, 406 (2006).

[11] M. W. Zwierlein, A. Schirotzek, C. H. Schunck, and W. Ketterle, Science 311, 492 (2006).

[12] G. B. Partridge, W. Li, R. I. Kamar, Y. A. Liao, and R. G. Hulet, Science 311, 503 (2006).

[13] M. W. Zwierlein, H. Schunck, A. Schirotzek, and W. Ketterle, cond-mat/0605258 (2006).

[14] E. Gubankova, A. Schmitt, and F. Wilczek, e-print cond-mat/0603603

[15] W. V. Liu and F. Wilczek, Phys. Rev. Lett. 90, 047002 (2003).

[16] M. M. Forbes, E. Gubankova, W. V. Liu, and F. Wilczek, Phys. Rev. Lett. 94, 017001 (2005).

[17] D. M. Eagles, Phys. Rev. 186, 456 (1969).

[18] A. J. Leggett, in Modern Trends in the Theory of Condensed Matter, edited by A. Pekalski and J. Przystawa (SpringerVerlag, Berlin, 1980), pp. 13-27.

[19] G. Sarma, J. Phys. Chem. Solids 24, 1029 (1963).

[20] H. Caldas, Phys. Rev. A 69, 063602 (2004).

[21] P. Fulde and R. A. Ferrell, Phys. Rev. 135, A550 (1964); A. I. Larkin and Y. N. Ovchinnikov, Zh. Exp. Teor. Fiz. 47, 1136 (1964) [Sov. Phys. JETP 20, 762 (1965)].

[22] D. Sheehy and L. Radzihovsky, Phys. Rev. Lett. 96, 060401
(2006).

[23] T. N. De Silva and E. J. Mueller, Phys. Rev. A 73, 051602(R) (2006).

[24] M. Haque and H. T. C. Stoof, cond-mat/0601321 (2006).

[25] J. Kinnunen, L. M. Jensen, and P. Torma, Phy. Rev. Lett. 96, 110403 (2006).

[26] L. Y. He, M. Jin, and P. F. Zhuang, eprint cond-mat/0606322

[27] C. H. Pao, S. T. Wu, and S. K. Yip, Phys. Rev. B 73, 132506 (2006).

[28] P. Pieri and G. C. Strinati, Phys. Rev. Lett. 96, 150404 (2006).

[29] K. Machida, T. Mizushima, and M. Ichioka, cond-mat/0604339 (2006).

[30] W. Yi and L. M. Duan, Phys. Rev. A 73, 031604(R) (2006).

[31] K. B. Gubbels, M. W. J. Romans, and H. T. C. S. H. T. C. Stoof, e-print cond-mat/0606330

[32] R. Casalbuoni and G. Nardulli, Rev. Mod. Phys. 76, 263 (2004).

[33] C.-C. Chien, Q. J. Chen, Y. He, and K. Levin, e-print condmat/0605039; Phys. Rev. Lett. 97, in production.

[34] C.-C. Chien, Q. J. Chen, Y. He, and K. Levin, Phys. Rev. A 74, 021602(R) (2006).

[35] A. Sedrakian and U. Lombardo, Phys. Rev. Lett. 84, 602 (2000).

[36] Q. J. Chen, H. Yan, C.-C. Chien, and K. Levin, e-print cond-mat/0608454

[37] P. Nozières and S. Schmitt-Rink, J. Low Temp. Phys. 59, 195 (1985).

[38] J. W. Serene, Phys. Rev. B 40, 10873 (1989).

[39] A. Perali, P. Pieri, and G. C. Strinati, Phys. Rev. Lett. 93, 100404 (2004).

[40] P. Pieri and G. C. Strinati, Phys. Rev. B. 71, 094520 (2005).

[41] I. Kosztin, Q. J. Chen, Y.-J. Kao, and K. Levin, Phys. Rev. B 61, 11662 (2000).

[42] J. Stajic, A. Iyengar, Q. J. Chen, and K. Levin, Phys. Rev. B 68, 174517 (2003).

[43] Q. J. Chen, I. Kosztin, B. Jankó, and K. Levin, Phys. Rev. Lett. 81, 4708 (1998).

[44] A. L. Fetter and J. D. Walecka, Quantum Theory of ManyParticle Systems (McGraw-Hill, San Francisco, 1971).

[45] J. Maly, B. Jankó, and K. Levin, Physica C 321, 113 (1999). 